\title{
A general approach to multivariable recursive interpolation
}

\section{A. Gombani ${ }^{1}$ (D) Gy. Michaletzky² ${ }^{2}$}

Received: 20 May 2019 / Accepted: 24 November 2020 / Published online: 28 January 2021

(C) The Author(s) 2021

\begin{abstract}
We consider here the problem of constructing a general recursive algorithm to interpolate a given set of data with a rational function. While many algorithms of this kind already exist, they are either providing non-minimal degree solutions (like the Schur algorithm) or exhibit jumps in the degree of the interpolants (or of the partial realization, as the problem is called when the interpolation is at infinity, see Rissanen (SIAM J Control 9(3):420-430, 1971) and Gragg and Lindquist (in: Linear systems and control (special issue), linear algebra and its applications, vol 50. pp 277-319, 1983)). By imbedding the solution into a larger set of interpolants, we show that the increase in the degree of this representation is proportional to the increase in the length of the data. We provide an algorithm to interpolate multivariable tangential sets of data with arbitrary nodes, generalizing in a fundamental manner the results of Kuijper (Syst Control Lett 31:225-233, 1997). We use this new approach to discuss a special scalar case in detail. When the interpolation data are obtained from the Taylor-series expansion of a given function, then the Euclidean-type algorithm plays an important role.
\end{abstract}

Keywords Interpolation $\cdot$ Recursion $\cdot$ Multivariable $\cdot$ Tangential

Mathematics Subject Classification 41A05 - 47A56 · 93B05 · 93D51

\section{Introduction}

The concepts of controllability indexes and tools from module theory like polynomial basis have been widely used in system theory and they have been extensively applied to different representations of transfer functions (rational functions, quotient

\footnotetext{
Gy. Michaletzky

michaletzky@caesar.elte.hu

A. Gombani gombani@ieiit.cnr.it

1 IEIIT-CNR, Via Giovanni Gradenigo 6a, 35131 Padua, Italy

2 Eötvös Loránd University, Pázmány Péter sétány 1/C, Budapest 1117, Hungary
} 
of polynomials, etc., see Kailath [12]), and in Antoulas et al. [3] they have also been connected to an interpolation problem for given data by means of a rational function. In [18], the present authors developed an approach for the characterizations-under weaker conditions - of proper interpolants by means of rational functions using controllability indexes. We show here how this framework accommodates quite naturally a recursive algorithm to compute the interpolants.

The simplest (and well-known) instance of the problem is, given a sequence of complex values $v_{0}, \ldots, v_{K}$, to find, for $k$ such that $0 \leq k \leq K$, all rational functions which interpolate those data at 0 , that is, functions of the form $f(s)=\sum_{i=0}^{k} s^{i} v_{i}+s^{k+1} g(s)$. This problem has a long history which (in its conceptual framework) goes back at least to Euler (see Wyman and Wyman [21] for a nice translation of Euler's paper and Meijering [17] for an exhaustive history of the problem) and, in its recursive form, has been widely studied especially in the case where there is one interpolation node at infinity (instead of 0: it is the well-known partial realization problem). In this context particularly relevant are the works of Ho and Kalman [11], of Rissanen [19] and of Gragg and Lindquist [10]. The common feature of all these algorithms is the intrinsic presence of sudden jumps in the degree of the interpolants as we add further data. A simple example of this behavior is a Fibonacci-like sequence which suddenly gets out of the recursion, like 1, 1, 2, 3, 5, 8, 13, 100: it can be seen that the degree of the recursion is 2 until it "hits" 100 and jumps to 4 . This makes these algorithms quite unsuitable for parameterization and thus for applications in identification. Their reliance on the Euclidean algorithm also makes extensions to the multivariable case or to different interpolation nodes intrinsically very difficult. The generalization of the approach initiated by Loewner [15] in 1934 for scalar interpolation problem was first systematically applied to the multivariable situation in Anderson and Antoulas [1]. The so-called Loewner matrices (divided difference matrices, null-pole coupling matrices) play a key tool in Ball et al. [4], Mayo and Antoulas [16] and Lefteriu and Antoulas [14], mentioning only a few. While the multivariable multiple node problem has been studied by several authors (see, for example, Antoulas et al. [3], Ball et al. [4] and Gombani and Michaletzky [9] for Kimura-Georgiou like fixed degree interpolants), a recursive version of these characterizations are-in general—not discussed. A recursive algorithm, which only works for two-sided interpolation, is presented for the matrix case in Lefteriu and Antoulas [14]. We provide here a recursive scheme under weaker conditions than those required in Antoulas et al. [3] and Fuhrmann [8].

We show how considering the larger (quite canonical) family of solutions (actually an immersion into a larger space) considered in [18], provides an algorithm which exhibits an astonishing regularity and makes the extensions to different nodes and the multivariable case quite straightforward. This provides a substantial improvement on Kuijper [13], where a single pole at infinity is considered and coprimeness assumption on the interpolating polynomials had to be made. This feature thus sparks renewed attention for the Behaviors approach to Systems Theory devised by Polderman and Willems [20], where lack of coprimeness of an AR representation was a fundamental and interesting feature of the models, but where the practical construction of recursive algorithms for such models relied on this very coprimeness assumption. Closer to our approach are the nice results in Boros et al. [5], where also polynomial families of solutions with the same regularity are obtained. Nevertheless, that approach does 
not guarantee uniqueness of the representation and its computation, in the case of confluent interpolation, is quite involved.

The fundamental idea is quite simple: most recursive techniques consider, for the scalar case, the smallest number for which some columns of data (in general arranged as a Hankel matrix) are linearly dependent. This means to consider enough elements so that we obtain a matrix with a one-dimensional kernel at each step. Adding a new row of data usually makes the matrix full rank and we thus have to add another column to this matrix to obtain a new kernel. The problems occur when the rank is not increased by the extra data for a while (the model already accommodates the new data) and then suddenly there is a jump in the rank. (We have to add several columns at once to have a nontrivial kernel.) We consider instead a slightly larger matrix, with a twodimensional kernel, for which we introduce an algorithm which always decreases by one the dimension of the kernel as we add data and thus has no jumps.

The paper is structured as follows: in Sect. 2 we introduce some general results for the tangential problem (see Antoulas et al. [3] or Ball et al. [4]) and discuss the existence of a polynomial solution. Since, as we said, the idea is quite simple, but the details for the multivariable case are rather intricate, in Sect. 3 we discuss the scalar rational interpolation problem and we lay the ground for characterizing all interpolants through the construction of a fat matrix (essentially a basis obtained from a nice selection with the addition of two extra columns) and connect it to a $2 \times 2$ polynomial matrix (see Antoulas et al. [3]) which we call fundamental solution: its main feature is that its rank is always 1 at the interpolation nodes and 2 everywhere else. (This solution is not unique, but all solutions are related by units.) As similar results are needed for the multivariable case, most proofs are deferred to that part. In Sect. 4 we exhibit a scalar interpolation algorithm in some detail, as we feel that it provides the essential ingredients for the multivariable case while providing a grasp of what the main idea is. In Sect. 5, we extend the construction of Sect. 3 to the case of tangential interpolating conditions. In Sect. 6 we show how to extend the recursive algorithm to the general tangential rational interpolation problem with arbitrary nodes. In Sect. 7 we apply our analysis to a scalar case having only one interpolation point at zero, and show how this special feature implies a very fine and detailed structure. In the "Appendix" we provide an example with the Fibonacci sequence.

Remark 1 The present approach seeks minimal degree interpolants and does not consider constrains on their norm and their analyticity, like in Nevanlinna-Pick or Caratheodory problems (see, for example, Dym [6] and Ball et al. [4]). It turns out, though that a similar, albeit non recursive, approach, can be used to tackle this kind of problems and that it yields a simpler solution to them. This will be discussed in a forthcoming paper.

\section{A general tangential interpolation problem}

Suppose that we are given a triplet $(\mathcal{A}, U, V)$, where $\mathcal{A}, U, V$ are of size $(K+1) \times$ $(K+1),(K+1) \times m$ and $(K+1) \times p$, respectively, determining a so-called tangential interpolation problem. The ultimate goal is to characterize all matrix-valued rational 
functions $Q(s)$ of size $m \times p$ possibly in the form $Q(s)=\beta(s) \alpha(s)^{-1}$ of a given McMillan degree (if any exists) such that

$$
(s I-\mathcal{A})^{-1}[U Q(s)-V]
$$

is analytic at $\sigma(\mathcal{A})$, the spectrum of $\mathcal{A}$.

Note that since the product $Q(s) \alpha(s)=\beta(s)$ is a polynomial a slightly different formulation of this problem is the following:

Problem 1 For a given triplet $(\mathcal{A}, U, V)$ find all the pairs of matrix polynomials $(\alpha, \beta)$ such that

$$
N(s):=(s I-\mathcal{A})^{-1}[U \beta(s)-V \alpha(s)]
$$

is a polynomial.

While the row dimensions of $\alpha$ and $\beta$ are $m$ and $p$, and their common column dimension could be an arbitrary natural number, it turns out that, to characterize all such pairs, we will need precisely $\alpha$ to be square and invertible.

Remark 2 Let us point out that, if $\beta$ and $\alpha$ are right coprime and $\alpha$ is an invertible polynomial matrix, then Problem 1 is equivalent to the following: set $Q(s)=\beta(s) \alpha^{-1}(s)$. There exist a polynomial matrix $p(s)$ such that

$$
\left((s I-\mathcal{A})^{-1} U+p(s)\right) Q(s)-(s I-\mathcal{A})^{-1} V
$$

is analytic on $\sigma(\mathcal{A})$.

Proof In fact, if $N(s)$ in Eq. (2) above is a matrix polynomial then the right coprime property of $\beta(s), \alpha(s)$ implies that there exist matrix-valued polynomials $\phi(s), \psi(s)$ such that

$$
N(s)=\phi(s) \beta(s)+\psi(s) \alpha(s) .
$$

It follows that $N(s) \alpha^{-1}(s)=\phi(s) Q(s)+\psi(s)$. Thus

$$
\left((s I-\mathcal{A})^{-1} U-\phi(s)\right) Q(s)-(s I-\mathcal{A})^{-1} V=N(s) \alpha^{-1}(s)-\phi(s) Q(s)=\psi(s),
$$

which is analytic on $\sigma(\mathcal{A})$.

The converse statement is obvious.

As it is well known from the literature on interpolation (see, e.g., [3,4]) a coprime factorization of $(s I-\mathcal{A})^{-1}[U,-V]$ plays a crucial role. Since for the derivation of the present results - except in Proposition 1, for the time being the distinction between the tangential conditions $U$ and the data $V$ is irrelevant, we will set, from now on, 
$W:=[U,-V]$ of dimension $(K+1) \times r$ and therefore we will consider the following polynomial coprime factorization

$$
(s I-\mathcal{A})^{-1} W=\Phi(s) \Gamma^{-1}(s)
$$

with $\Phi, \Gamma$ right coprime. We will also refer to the interpolation problem as one defined by $(\mathcal{A}, W)$ instead of $(\mathcal{A}, U, V)$. Thus, clearly (3) can also be written as

$$
(s I-\mathcal{A}) \Phi(s)=W \Gamma(s)
$$

provided that $\Phi$ and $\Gamma$ are right coprime and $\Gamma(s)$ is invertible.

The following simple lemma—see Ex. 6.3-19 in Kailath [12]—provides a straightforward way to check whether, for a given factorization, this is the case:

Lemma 1 Assume that the pair $(\mathcal{A}, W)$ is controllable. Then, if the degree $\operatorname{deg} \operatorname{det} \Gamma=$ $\operatorname{deg} \operatorname{det}(s I-\mathcal{A})=K+1$, then $\Phi$ and $\Gamma$ in (4) are right coprime.

We will assume, from now on, that $\Phi$ and $\Gamma$ are right coprime. There are a few properties of (3) which will be needed.

Remark 3 Since $\Phi, \Gamma$ are right coprime, $(s I-\mathcal{A})$ and $\Gamma(s)$ have the same-nonunity-invariant factors; especially, $\operatorname{det} \Gamma(s)=\operatorname{det}(s I-\mathcal{A})$, and thus, the zeros of $\operatorname{det} \Gamma$ are contained in $\sigma(\mathcal{A})$. Coprimeness also implies that if $\Gamma(\lambda) \xi=0$ for some vector $\xi \in \mathbb{C}^{r}$, and $\lambda \in \mathbb{C}$, then $\Phi(\lambda) \xi \neq 0$; therefore, $\Phi(\lambda) \xi$ is in the kernel of $(\lambda I-\mathcal{A})$.

With some abuse of notation, we say that a polynomial matrix $\gamma$ generates solutions to Problem 1 defined by $(\mathcal{A}, W)$ if there exists a polynomial matrix $\phi$ such that

$$
(s I-\mathcal{A}) \phi(s)=W \gamma(s) .
$$

In other words, using $\gamma(s)$ as a polynomial input to the transfer function $(s I-\mathcal{A})^{-1} W$ the output- $\phi(s)$ - is polynomial, as well.

Although the next lemma is essentially the basic starting block of the derivation of all solutions to an interpolation problem in the form of linear fractional transformation (cf. $[3,4,14])$ for the readers convenience we include here a short proof of it.

Lemma 2 Assume that the pair $(\mathcal{A}, W)$ is controllable and $\gamma$ generates a solution to Problem 1 defined by $(\mathcal{A}, W)$. Then, there exists a matrix polynomial $\pi(s)$ such that $\gamma(s)=\Gamma(s) \pi(s)$ (that is, $\Gamma(s)$ generates the module of polynomials solving Problem 1).

Proof The pair $(\mathcal{A}, W)$ is controllable. Thus, $(s I-\mathcal{A})$ and $W$ are left coprime and according to our assumptions $\Phi, \Gamma$ in (4) are right coprime. Therefore-using Lemma 6.4-2 in [12]—there exist matrix polynomials $X(s), Y(s), \bar{X}(s), \bar{Y}(s)$ such that

$$
\left[\begin{array}{cc}
(s I-\mathcal{A}) & W \\
\bar{X}(s) & -\bar{Y}(s)
\end{array}\right]\left[\begin{array}{cc}
X(s) & \Phi(s) \\
Y(s) & -\Gamma(s)
\end{array}\right]=\left[\begin{array}{ll}
I & 0 \\
0 & I
\end{array}\right]
$$


Thus we can always write

$$
\left[\begin{array}{c}
-\phi(s) \\
\gamma(s)
\end{array}\right]=\left[\begin{array}{cc}
X(s) & \Phi(s) \\
Y(s) & -\Gamma(s)
\end{array}\right]\left[\begin{array}{cc}
(s I-\mathcal{A}) & W \\
\bar{X}(s) & -\bar{Y}(s)
\end{array}\right]\left[\begin{array}{c}
-\phi(s) \\
\gamma(s)
\end{array}\right]
$$

If $\phi(s), \gamma(s)$ satisfy (5), we obtain

$$
\left[\begin{array}{c}
-\phi(s) \\
\gamma(s)
\end{array}\right]=\left[\begin{array}{cc}
X(s) & \Phi(s) \\
Y(s) & -\Gamma(s)
\end{array}\right]\left[\begin{array}{c}
0 \\
-(\bar{X}(s) \phi(s)+\bar{Y} \gamma(s))
\end{array}\right],
$$

that is, setting $\pi(s):=\bar{X}(s) \phi(s)+\bar{Y}(s) \gamma(s)$, we obtain $\gamma(s)=\Gamma(s) \pi(s)$, as wanted.

The following proposition shows that under some mild conditions there exists a matrix polynomial solution to Problem 1.

Proposition 1 Consider Problem 1 determined by the triplet $(\mathcal{A}, U, V)$ assuming that the pair $(\mathcal{A},[U, V])$ is controllable.

Then there exists a matrix polynomial $\beta_{0}(s)$ such that $\gamma_{0}(s)=\left[\begin{array}{c}\beta_{0} \\ I\end{array}\right]$ provides a solution to Problem 1 if and only if the pair $(\mathcal{A}, U)$ is controllable. (That is in this case $Q(s)=\beta_{0}(s) I^{-1}=\beta_{0}(s)$ is a polynomial matrix. )

Proof Consider the coprime factorization of $(s I-\mathcal{A})^{-1}[U,-V]$ defined in (3) and define a partitioned form of $\Gamma$ :

$$
\left[\begin{array}{ll}
\beta^{U}(s) & \beta^{V}(s) \\
\alpha^{U}(s) & \alpha^{V}(s)
\end{array}\right]
$$

corresponding to the partition $W=[U,-V]$.

First we show the controllability of the pair $(\mathcal{A}, U)$ is equivalent to the left coprimeness of $\alpha^{U}$ and $\alpha^{V}$. Assume that there exists a scalar $s_{0} \in \mathbb{C}$ such that the matrix $\left[\alpha^{U}\left(s_{0}\right), \alpha^{V}\left(s_{0}\right)\right]$ is not of full row-rank. This implies that there exists a vector $\eta$ such that

$$
\eta^{*}\left[\alpha^{U}\left(s_{0}\right), \alpha^{V}\left(s_{0}\right)\right]=0
$$

and the matrix $\Gamma\left(s_{0}\right)$ is singular. In particular,

$$
\left[0, \eta^{*}\right] \Gamma\left(s_{0}\right)=0
$$

At the same time, according to Remark 3 the zeros of det $\Gamma(s)$ are contained in $\sigma(\mathcal{A})$ thus $s_{0}$ is necessarily an eigenvalue of $\mathcal{A}$. Since according again to Remark 3 the nonunity invariant factors of $s I-\mathcal{A}$ and $\Gamma(s)$ coincide thus the dimensions of the left kernel of $s_{0} I-\mathcal{A}$ and that of $\Gamma\left(s_{0}\right)$ are equal. Let us observe that the controllability of the pair $(\mathcal{A},[U,-V])$ implies taking a basis in the subspace of the left eigenvectors of $\mathcal{A}$ with eigenvalue $s_{0}$ their image vectors under the transformation defined by $[U,-V]$ 
should be also linearly independent-otherwise there would exist a left eigenvector of $\mathcal{A}$ orthogonal to the columns of $[U,-V]$ contradicting to the controllability of $(\mathcal{A},[U,-V])$. Since the dimensions of the left kernels of $s_{0} I-\mathcal{A}$ and $\Gamma\left(s_{0}\right)$ coincide and the vector $\left[0, \eta^{*}\right]$ is in the left kernel of $\Gamma\left(s_{0}\right)$ there exists a vector $\xi$ such that

$$
\begin{aligned}
\xi^{*}\left(s_{0} I-\mathcal{A}\right) & =0, \\
\xi^{*}[U,-V] & =\left[0, \eta^{*}\right] .
\end{aligned}
$$

In particular, $\xi^{*} U=0$. Thus, the pair $(\mathcal{A}, U)$ is not a controllable pair.

Conversely, if the pair $(\mathcal{A}, U)$ in not controllable, then there exists a left eigenvector $\xi$ of $\mathcal{A}$ for which $\xi^{*} U=0$. Denoting the corresponding eigenvalue by $s_{0}$ we get that $\xi^{*}\left(s_{0} I-\mathcal{A}\right)=0$, implying that

$$
0=\xi^{*}\left(s_{0} I-\mathcal{A}\right) \Phi\left(s_{0}\right)=\xi^{*}[U,-V] \Gamma\left(s_{0}\right)=-\xi^{*} V\left[\alpha^{U}\left(s_{0}\right), \alpha^{V}\left(s_{0}\right)\right] \text {. }
$$

Since the vector $\xi^{*}[U,-V]$ is nonzero due to the controllability of the pair $(\mathcal{A},[U,-V])$ we have that $\xi^{*} V \neq 0$. This implies that the polynomial matrices $\left[\alpha^{U}\right.$ and $\alpha^{V}$ are not left coprime.

Next we show that the left coprimeness of $\alpha^{U}$ and $\alpha^{V}$ is equivalent to the existence of a matrix polynomial $\beta_{0}$ such that $\left(I, \beta_{0}\right)$ provides a solution to Problem 1 .

Since according to Lemma 2 any solution can be written in the form $\Gamma \pi$ thus if $\left(I, \beta_{0}\right)$ provides a solution then for some matrix polynomial $\pi$ we have that

$$
\Gamma \pi=\left[\begin{array}{cc}
\beta^{U}(s) & \beta^{V}(s) \\
\alpha^{U}(s) & \alpha^{V}(s)
\end{array}\right]\left[\begin{array}{l}
\pi^{U}(s) \\
\pi^{V}(s)
\end{array}\right]=\left[\begin{array}{c}
\beta_{0} \\
I
\end{array}\right] .
$$

The second entry shows that $\alpha^{U}$ and $\alpha^{V}$ are left coprime.

Conversely, if $\alpha^{U}, \alpha^{V}$ are left coprime, then there exist a polynomial pair $\pi^{U}, \pi^{V}$ such that

$$
\left[\alpha^{U}(s), \alpha^{V}(s)\right]\left[\begin{array}{l}
\pi^{U}(s) \\
\pi^{V}(s)
\end{array}\right]=I .
$$

Introducing the notation

$$
\pi(s)=\left[\begin{array}{l}
\pi^{U}(s) \\
\pi^{V}(s)
\end{array}\right]
$$

and

$$
\beta_{0}(s)=\left[\beta^{U}(s), \beta^{V}(s)\right] \pi(s) .
$$

We obtain that

$$
\left[\begin{array}{c}
\beta_{0}(s) \\
I
\end{array}\right]=\Gamma(s) \pi(s)
$$


Thus $\left[\begin{array}{c}\beta_{0} \\ I\end{array}\right]$ provides a solution to Problem 1, concluding the proof of the proposition.

Remark 4 Let us point out the following immediate corollary of the previous Lemma. If the pair $(\mathcal{A}, U)$ is controllable, then the rank drop of the polynomial matrix $\Gamma(s)$ cannot be greater than the number of the columns of $U$ at any $s \in \mathbb{C}$.

The following proposition slightly generalizes the argument applied in the proof of Theorem 3.5 in [3].

Proposition 2 Consider Problem 1 determined by the triplet $(\mathcal{A}, U, V)$ assuming that the pair $(\mathcal{A}, U)$ is controllable. According to the previous Proposition there exists a polynomial solution $\left[\begin{array}{c}\beta_{0} \\ I\end{array}\right]$. Let us consider a right coprime factorization of (s I $\mathcal{A})^{-1} U=\Psi_{1}(s) \Gamma_{1}(s)^{-1}$.

Then all solutions $\left[\begin{array}{l}\beta(s) \\ \alpha(s)\end{array}\right]$ can be written in the form

$$
\left[\begin{array}{c}
\beta(s) \\
\alpha(s)
\end{array}\right]=\left[\begin{array}{c}
\Gamma_{1}(s) \pi_{g}(s)+\beta_{0}(s) \alpha(s) \\
\alpha(s)
\end{array}\right]
$$

for some matrix polynomial $\pi_{g}(s)$.

In particular, in this case

$$
Q(s)=\beta(s) \alpha^{-1}(s)=\Gamma_{1}(s) \pi_{g}(s) \alpha^{-1}(s)+\beta_{0}(s),
$$

where $\beta_{0}(s)$ is a particular polynomial solution (might be called Hermite interpolant), and $(s I-\mathcal{A})^{-1} U \Gamma_{1}(s)=\Psi_{1}(s)$ is already a polynomial matrix.

Proof According to Proposition 1 there exists a polynomial matrix $\pi_{0}$ such that $\left[\begin{array}{c}\beta_{0}(s) \\ I\end{array}\right]=\Gamma(s) \pi_{0}(s)$. The right coprime factorization of $(s I-\mathcal{A})^{-1} U=$ $\Psi_{1}(s) \Gamma_{1}(s)^{-1}$ can be written as

$$
(s I-\mathcal{A})^{-1} U \Gamma_{1}(s)=(s I-\mathcal{A})^{-1}[U,-V]\left[\begin{array}{c}
\Gamma_{1}(s) \\
0
\end{array}\right]=\Psi_{1}(s)
$$

which is a polynomial; thus, Lemma 2 implies that $\left[\begin{array}{c}\Gamma_{1}(s) \\ 0\end{array}\right]=\Gamma(s) \pi_{1}(s)$ for some polynomial $\pi_{1}(s)$. In other words

$$
\left[\begin{array}{cc}
\Gamma_{1}(s) & \beta_{0}(s) \\
0 & I
\end{array}\right]=\Gamma(s)\left[\pi_{1}(s), \pi_{0}(s)\right]
$$

Since both $\Gamma$ and $\Gamma_{1}$ were defined by coprime factorizations, we have that $\operatorname{det}(s I-$ $\mathcal{A})=\operatorname{det} \Gamma(s)=\operatorname{det} \Gamma_{1}(s)$. Consequently, $\operatorname{det}\left[\pi_{1}(s), \pi_{0}(s)\right]=1$, that is, it has a 
polynomial inverse. Thus-using Lemma 2-if $\gamma(s)=\left[\begin{array}{c}\beta(s) \\ \alpha(s)\end{array}\right]$ generates solutions

to Problem 1 then there exists a matrix polynomial $\pi(s)=\left[\begin{array}{l}\pi_{g}(s) \\ \pi_{d}(s)\end{array}\right]$ such that

$$
\left[\begin{array}{l}
\beta(s) \\
\alpha(s)
\end{array}\right]=\left[\begin{array}{cc}
\Gamma_{1}(s) & \beta_{0}(s) \\
0 & I
\end{array}\right]\left[\begin{array}{l}
\pi_{g}(s) \\
\pi_{d}(s)
\end{array}\right]
$$

concluding the proof of the remark.

Although all the ingredients of the recursive algorithm for constructing solutions of the tangential interpolation problem are quite simple, the details for the multivariate case are rather cumbersome, and therefore, we start our analysis with the scalar casepresenting the basic ideas in a simpler form and reverting to the general multivariate case in Sect. 5. But in order to avoid repetitions the detailed proofs will be given for the matrix-valued functions.

\section{The template problem: scalar interpolation}

We consider the situation when all the interpolation nodes are at the origin. Assume that we are given the data $\left\{v_{0}, v_{1}, \ldots, v_{K}\right\}$ and want to characterize all rational functions $Q(s)=\frac{\beta(s)}{\alpha(s)}$ of a given degree (if any exists) such that

$$
Q(s)=\frac{\beta(s)}{\alpha(s)}=\sum_{i=0}^{K} v_{i} s^{i}+s^{K+1} Q_{1}(s),
$$

where $Q_{1}(s)$ is a rational function analytic in 0 . We do not presently require that $Q(s)$ is proper: a method to achieve this condition is thoroughly examined in [18].

Note that the product $\alpha(s) Q_{1}(s)$ should obviously be a polynomial. Thus a slightly different formulation of the problem is the following.

Problem 2 Find all the pairs $(\alpha, \beta)$ such that

$$
\frac{1}{s^{K+1}}\left(\beta(s)-\alpha(s) \sum_{i=0}^{K} v_{i} s^{i}\right) .
$$

is a polynomial.

(These two formulations coincide if $\alpha$ and $\beta$ are coprime).

A second well-known instance is, given points $\lambda_{0}, \ldots \lambda_{K} \in \mathbb{C}$ distinct, and values $v_{0}, \ldots v_{K}$, to find all rational functions $Q(s)$ of a given degree (if any exists) such that

$$
Q\left(\lambda_{i}\right)=\frac{\beta\left(\lambda_{i}\right)}{\alpha\left(\lambda_{i}\right)}=v_{i} \quad i=0, \ldots K
$$

Again, if we assume $\alpha$ and $\beta$ coprime, we can rewrite the problem as 
Problem 3 Find all the coprime pairs $(\alpha, \beta)$ such that

$$
\frac{1}{s-\lambda_{i}}\left[\beta(s)-\alpha(s) v_{i}\right]
$$

is a polynomial for all $i: 0 \leq i \leq K$.

Both problems can be written in the same matricial form: in case of (9) define:

$$
\mathcal{A}=\left[\begin{array}{cccc}
0 & \ldots & 0 & 0 \\
1 & 0 & \vdots & 0 \\
& \ddots & 0 & \vdots \\
0 & \ldots & 1 & 0
\end{array}\right], \quad U=\left[\begin{array}{c}
1 \\
0 \\
\vdots \\
0
\end{array}\right], \quad V=\left[\begin{array}{c}
v_{0} \\
v_{1} \\
\vdots \\
v_{K}
\end{array}\right]
$$

where $\mathcal{A}$ is a $(K+1) \times(K+1)$ matrix and $U, V$ are $(K+1)$-dimensional vectors. For (11), set $\mathcal{A}:=\operatorname{diag}\left\{\lambda_{1}, \ldots, \lambda_{K}\right\}, U:=[1,1, \ldots, 1]^{T}$ and $V$ as above.

Both problems are thus immediately seen to be special cases of the

Problem 4 Find all the pairs $(\alpha, \beta)$ such that $G(s)$

$$
G(s)=(s I-\mathcal{A})^{-1}(U \beta(s)-V \alpha(s)) \text { is a polynomial vector. }
$$

We will thus focus on the general case (which includes the above examples) of a given matrix $\mathcal{A}$ of dimension $(K+1) \times(K+1)$ and vectors $U, V$ of dimension $K+1$.

We will assume that the pair $(\mathcal{A}, U)$ is controllable and, to construct recursively the interpolants, that the matrix $\mathcal{A}$ is lower triangular.

Since the recursive algorithm will be formulated using the coefficients of the pair $\alpha, \beta$ we need an equivalent formulation of Problem 4 .

We introduce the following notation: for any polynomial $\gamma$ we denote by $\gamma$ the column vector of the coefficients — starting with the constant term-of the polynomial $\gamma$. (In some cases we will have to increase the dimension of the corresponding vector, by adding extra zeros as entries. This is in coherence with considering higher order terms in the polynomial but with zero coefficients.) In case of the polynomials $\beta^{U}(s), \beta^{V}(s), \alpha^{U}(s), \alpha^{V}(s)$ we shall use $\boldsymbol{\beta}^{U}, \boldsymbol{\beta}^{V}, \boldsymbol{\alpha}^{U}, \boldsymbol{\alpha}^{V}$ for the corresponding column vectors.

We set $U^{j}$ to be the matrix of dimension $(K+1) \times(j+1)$ of the form

$$
U^{j}=\left[U, \mathcal{A} U, \mathcal{A}^{2} U, \ldots, \mathcal{A}^{j} U\right]
$$

Similarly, we set $V^{r}$ to be the matrix of dimension $(K+1) \times(r+1)$ of the form

$$
V^{r}=\left[V, \mathcal{A} V, \mathcal{A}^{2} V, \ldots, \mathcal{A}^{r} V\right]
$$

Notice that $U=U^{0}$ and $V=V^{0}$. 
Lemma 3 Let $\alpha=\sum_{i} \alpha_{i} s^{i}, \beta=\sum_{i} \beta_{i} s^{i}$ be two polynomials. Consider integers $j, r$ for which $j \geq \operatorname{deg} \beta, r \geq \operatorname{deg} \alpha$. Then condition (13) is equivalent to

$$
U^{j} \boldsymbol{\beta}=V^{r} \boldsymbol{\alpha} .
$$

Moreover, if $\alpha, \beta$ have a common factor $s-\lambda_{0}$, that is $\alpha(s)=\left(s-\lambda_{0}\right) \alpha_{1}(s)$ and $\beta(s)=\left(s-\lambda_{0}\right) \beta_{1}(s)$, with $\lambda_{0} \notin \sigma(\mathcal{A})$, then $U^{j-1} \boldsymbol{\beta}_{1}=V^{r-1} \boldsymbol{\alpha}_{1}$.

The proof is deferred to the general case result in Theorem 2

Definition 1 We say that $\left[\begin{array}{ll}\beta^{U} & \beta^{V} \\ \alpha^{U} & \alpha^{V}\end{array}\right]$ is a fundamental solution to Problem 4 determined by the data $(\mathcal{A}, U, V)$ if each of its columns are solutions to the Problem 4 and for any other solution $(\beta, \alpha)$, there exist polynomials $\pi^{U}, \pi^{V}$ such that

$$
\left[\begin{array}{l}
\beta \\
\alpha
\end{array}\right]=\left[\begin{array}{ll}
\beta^{U} & \beta^{V} \\
\alpha^{U} & \alpha^{V}
\end{array}\right]\left[\begin{array}{l}
\pi^{U} \\
\pi^{V}
\end{array}\right]
$$

We saw in Lemma 2 that, if $\Psi(s), \Gamma(s)$ are coprime, $\Gamma(s)$ is a fundamental solution.

To construct a coprime factorization (3) from the data, we denote by $\mu$ and $\nu$ the controllability indexes of $U$ and $V$ relatively to $(\mathcal{A},[U, V])$. (That is, consider the vectors $U, V, \mathcal{A} U, \mathcal{A} V, \mathcal{A}^{2} U, \mathcal{A}^{2} V, \ldots$ in this order. Then $\mu$ is the smallest number for which $\mathcal{A}^{\mu} U$ can be expressed as a linear combination of its preceding vectors. The $v$ is defined similarly.) In view of controllability, $\mu+v=K+1$. Then, for suitable coefficients $\alpha_{i}^{U}, \beta_{i}^{U}$ the identity

$$
\sum_{i=0}^{\mu} \beta_{i}^{U} \mathcal{A}^{i} U=\sum_{i=0}^{\mu-1} \alpha_{i}^{U} \mathcal{A}^{i} V
$$

is satisfied with $\beta_{\mu}^{U}=1$. Similarly, for suitable coefficients $\alpha_{i}^{V}, \beta_{i}^{V}$ the identity

$$
\sum_{i=0}^{v} \beta_{i}^{V} \mathcal{A}^{i} U=\sum_{i=0}^{v} \alpha_{i}^{V} \mathcal{A}^{i} V
$$

holds with $\alpha_{v}^{V}=1$.

Lemma 4 Let the polynomials $\alpha^{U}(s)$ and $\beta^{U}(s)$ be defined as

$$
\alpha^{U}(s)=\sum_{i=0}^{\mu-1} \alpha_{i}^{U} s^{i} \text { and } \beta^{U}(s)=\sum_{i=0}^{\mu} \beta_{i}^{U} s^{i}
$$

where the coefficients are those in (17); let $\alpha^{V}(s), \beta^{V}(s)$ be defined similarly from (18). Then

$$
\Gamma(s)=\left[\begin{array}{cc}
\beta^{U}(s) & \alpha^{V}(s) \\
\alpha^{U}(s) & \alpha^{V}(s)
\end{array}\right]
$$


is a fundamental solution to Problem 4.

Proof Let us point out that that for the polynomials constructed above the inequalities

$$
\operatorname{deg} \alpha^{U}(s)<\operatorname{deg} \beta^{U}(s), \quad \operatorname{deg} \beta^{V} \leq \alpha^{V}(s) .
$$

hold. Thus this restriction gives us a fundamental solution of special type, although it does not imply uniqueness. For a general fundamental solution these inequalities do not necessarily hold.

From Lemma 3 we obtain that the coefficients $\alpha_{i}^{U}, \alpha_{i}^{V}, \beta_{i}^{U}, \beta_{i}^{V}, i \geq 0$, define polynomials providing solutions to Problem 4 , that is there exists a matrix polynomial $\Psi(s)$ of size $(K+1) \times 2$ such that Eq. (3) holds. Since we can assume that $\beta^{U}(s)$ and $\alpha^{V}(s)$ are monic, the matrix of the coefficients of the highest column degrees of $\Gamma(s)$ is upper triangular, with identity on the diagonal. Since $\mu+v=K+1$, the degree of det $\Gamma(s)$ is $K+1$, that is it coincides with that of $\operatorname{det}(s I-\mathcal{A})$. Using (3), the controllability of the pair $(\mathcal{A},[U, V])$ gives in view of Ex. 6.3-19 in Kailath [12] that $\Psi(s)$ and $\Gamma(s)$ are right coprime.

In order to ensure uniqueness, introduce the following notion:

Definition 2 A minimal fundamental solution (MF solution) is a fundamental solution such that

$$
\begin{aligned}
& \operatorname{deg} \beta^{U}(s)=\mu, \quad \operatorname{deg} \alpha^{V}(s)=v, \quad \beta^{U}, \alpha^{V} \text { are monic } ; \\
& \operatorname{deg} \beta^{V}(s) \leq \min \left(\operatorname{deg} \beta^{U}(s)-1, \operatorname{deg} \alpha^{V}(s)\right) \\
& \operatorname{deg} \alpha^{U}(s)<\min \left(\operatorname{deg} \beta^{U}(s), \operatorname{deg} \alpha^{V}(s)\right) .
\end{aligned}
$$

Proposition 3 For any controllable set of data $(\mathcal{A}, U, V)$ there exists a unique $M F$ solution.

Proof the statement is essentially trivial because Lemmas 3 and 4 show that the MF solution is nothing else than a basis selection scheme. Another way to see this if, for example $\mu>v$, is that the vector $\mathcal{A}^{\mu} U$ can be uniquely expressed in terms of the columns of

$\left[U, V, \mathcal{A} U, \mathcal{A} V, \ldots, \mathcal{A}^{\nu-1} U, \mathcal{A}^{\nu-1} V, \mathcal{A}^{v} U, \ldots, \mathcal{A}^{\mu-1} U\right]$, since the matrix has full rank (similarly for $\mathcal{A}^{v} V$ ). For sake of completeness, we provide a proof in terms of matrices for the multivariable case in Proposition (4).

\section{Scalar recursive interpolation}

The interest of MF solutions is that its regularity and uniqueness allow for a straightforward recursion algorithm which, as we shall see, can be easily generalized to the multivariable case.

We consider the situation where we have a sequence of nested problems indexed by $k \leq K$, where $\mathcal{A}_{k}$ is lower triangular $(k+1) \times(k+1)$ and $U_{k}$ and $V_{k}$ are $(k+1)$ - 
dimensional vectors. We say that the problems are nested if, for each $k$,

$$
\mathcal{A}_{k+1}=\left[\begin{array}{cc}
\mathcal{A}_{k} & 0 \\
\boldsymbol{a}_{k+1} & \lambda_{k+1}
\end{array}\right], \quad U_{k+1}=\left[\begin{array}{c}
U_{k} \\
u_{k+1}
\end{array}\right], \quad V_{k+1}=\left[\begin{array}{c}
V_{k} \\
v_{k+1}
\end{array}\right]
$$

and the pair $\left(\mathcal{A}_{k}, U_{k}\right)$ is controllable for $k \leq K$. Our goal is to present a recursive algorithm to compute a minimal degree solution for each $k$ from the solution for $k-1$.

As in (3), for each $k$, we can consider right coprime polynomial matrices $\Gamma_{k}(s)$ and $\Psi_{k}(s)$ such that

$$
\left(s I-\mathcal{A}_{k}\right) \Psi_{k}(s)=\left[U_{k},-V_{k}\right] \Gamma_{k}(s) .
$$

Partitioning $\Gamma_{k}(s)$ as $\Gamma_{k}:=\left[\begin{array}{cc}\beta_{k}^{U} & \beta_{k}^{V} \\ \alpha_{k}^{U}, & \alpha_{k}^{V}\end{array}\right]$, the above Eq. (21) becomes

$$
\left(s I-\mathcal{A}_{k}\right) \Psi_{k}(s)=\left[U_{k},-V_{k}\right]\left[\begin{array}{cc}
\beta_{k}^{U} & \beta_{k}^{V} \\
\alpha_{k}^{U}, & \alpha_{k}^{V}
\end{array}\right]
$$

As in (14) and (15), we can define, for each $k, j, r$ integers, $U_{k}^{j}$ and $V_{k}^{r}$ as

$$
\begin{aligned}
U_{k}^{j} & =\left[U_{k}, \mathcal{A}_{k} U_{k}, \mathcal{A}_{k}^{2} U_{k}, \ldots, \mathcal{A}_{k}^{j} U_{k}\right], \\
V_{k}^{r} & =\left[V_{k}, \mathcal{A}_{k} V_{k}, \mathcal{A}_{k}^{2} V_{k}, \ldots, \mathcal{A}_{k}^{r} V_{k}\right] .
\end{aligned}
$$

Let $\left[\begin{array}{c}\beta_{k}^{U} \\ \alpha_{k}^{U}\end{array}\right]$ and $\left[\begin{array}{c}\beta_{k}^{V} \\ \alpha_{k}^{V}\end{array}\right]$ be the MF solution to the Problem 4 determined by the data $\left(\mathcal{A}_{k}, U_{k}, V_{k}\right)$ with controllability indexes $\mu_{k}$ and $v_{k}$, that is $\left[\begin{array}{c}\boldsymbol{\beta}_{k}^{U} \\ \boldsymbol{\alpha}_{k}^{U}\end{array}\right]$ and $\left[\begin{array}{c}\boldsymbol{\beta}_{k}^{V} \\ \boldsymbol{\alpha}_{k}^{V}\end{array}\right]$ span the kernel two-dimensional kernel of $\left[U_{k}^{\mu_{k}},-V_{k}^{\nu_{k}}\right]$.

The following remark contains the core idea of the recursion:

Remark 5 The matrix $\left[U_{k}^{\mu_{k}},-V_{k}^{\nu_{k}}\right]$ has $k+1$ rows (of data), $k+3$ columns and thus, in view of controllability, it has a two-dimensional kernel. The recursion adds one row to this matrix: controllability will imply that its kernel is always one dimensional. Since it must be contained in the previous kernel (in view of the triangularity of $\mathcal{A}$ ), it can be expressed as a linear combination of $\left[\begin{array}{c}\boldsymbol{\beta}_{k}^{U} \\ \boldsymbol{\alpha}_{k}^{U}\end{array}\right]$ and $\left[\begin{array}{c}\boldsymbol{\beta}_{k}^{V} \\ \boldsymbol{\alpha}_{k}^{V}\end{array}\right]$. This is the first step of the algorithm. To find the appropriate linear combinations the "error terms" should be considered arising from the solutions obtained in the $k^{\mathrm{k}}$ step but applying those for the next interpolation data. Let us note that the construction of a recursive scheme for simultaneous left and right interpolation in [14] using Loewner matrix pencils is also based on the use of similar error terms. 
The second step consists of adding a column to this extended matrix, so that its kernel has again dimension two and the property of being a minimal fundamental solution is preserved, as well.

In view of the above remark, us denote by $\left[u_{k+1}^{\mu_{k}},-v_{k+1}^{\nu_{k}}\right]$ (with components of dimension $\left(\mu_{k}+1\right)$ and $\left(v_{k}+1\right)$, respectively) the last row of the extended matrix $\left[U_{k+1}^{\mu_{k}},-V_{k+1}^{v_{k}}\right]$, that is

$$
U_{k+1}^{\mu_{k}}=\left[\begin{array}{c}
U_{k}^{\mu_{k}} \\
u_{k+1}^{\mu_{k}}
\end{array}\right], \quad V_{k+1}^{v_{k}}=\left[\begin{array}{c}
V_{k}^{\nu_{k}} \\
v_{k+1}^{\nu_{k}}
\end{array}\right]
$$

and compute the error terms arising from using the linear combinations obtained in the $k^{\text {th }}$ step for the next interpolation values $u_{k+1}, v_{k+1}$, as well:

$$
\begin{aligned}
& {\left[\epsilon_{k+1}^{U}, \epsilon_{k+1}^{V}\right]:=\left[u_{k+1}^{\mu_{k}},-v_{k+1}^{v_{k}}\right]\left[\begin{array}{cc}
\boldsymbol{\beta}_{k}^{U} & \boldsymbol{\beta}_{k}^{V} \\
\boldsymbol{\alpha}_{k}^{U} & \boldsymbol{\alpha}_{k}^{V}
\end{array}\right]} \\
& \quad=\left[\sum_{j=0}^{\mu_{k}}\left(\beta_{k, j}^{U} u_{k-j+1}-\alpha_{k, j}^{U} v_{k-j+1}\right), \sum_{j=0}^{v_{k}}\left(\beta_{k, j}^{V} u_{k-j+1}-\alpha_{k, j}^{V} v_{k-j+1}\right)\right] .
\end{aligned}
$$

Let us point out that if $\epsilon_{k+1}^{U}=0$ then $\mu_{k+1}=\mu_{k}$ while $\epsilon_{k+1}^{V}=0$ implies that $v_{k+1}=v_{k}$. In view of controllability, this implies that either $\epsilon_{k+1}^{U}$ or $\epsilon_{k+1}^{V}$ is different from zero. Furthermore, if $\mu_{k+1}=\mu_{k} \leq v_{k}$ then $\epsilon_{k+1}^{U}=0$ and similarly, if $v_{k+1}=$ $v_{k}<\mu_{k}$ then $\epsilon_{k+1}^{V}=0$.

Now, if $\epsilon_{k+1}^{U} \neq 0$, then

$$
\left[u_{k+1}^{\mu_{k}},-v_{k+1}^{v_{k}}\right]\left[\begin{array}{cc}
\boldsymbol{\beta}_{k}^{U} & \boldsymbol{\beta}_{k}^{V} \\
\boldsymbol{\alpha}_{k}^{U} & \boldsymbol{\alpha}_{k}^{V}
\end{array}\right]\left[-\frac{\epsilon_{k+1}^{V}}{\epsilon_{k+1}^{U}}\right]=\left[\epsilon_{k+1}^{U}, \epsilon_{k+1}^{V}\right]\left[\begin{array}{c}
-\frac{\epsilon_{k+1}^{V}}{\epsilon_{k+1}^{U}} \\
1
\end{array}\right]=0 .
$$

The above procedure allows to identify an element in the kernel of $\left[U_{k+1}^{\mu_{k}}, V_{k+1}^{\nu_{k}}\right]$, and, more precisely, if $\epsilon_{k+1}^{U} \neq 0$,

$$
\left[U_{k+1}^{\mu_{k}},-V_{k+1}^{\nu_{k}}\right]\left(-\frac{\epsilon_{k+1}^{V}}{\epsilon_{k+1}^{U}}\left[\begin{array}{c}
\boldsymbol{\beta}_{k}^{U} \\
\boldsymbol{\alpha}_{k}^{U}
\end{array}\right]+\left[\begin{array}{c}
\boldsymbol{\beta}_{k}^{V} \\
\boldsymbol{\alpha}_{k}^{V}
\end{array}\right]\right)=0
$$

Similarly, if $\epsilon_{k+1}^{V} \neq 0$,

$$
\left[U_{k+1}^{\mu_{k}},-V_{k+1}^{v_{k}}\right]\left(\left[\begin{array}{c}
\boldsymbol{\beta}_{k}^{U} \\
\boldsymbol{\alpha}_{k}^{U}
\end{array}\right]-\frac{\epsilon_{k+1}^{U}}{\epsilon_{k+1}^{V}}\left[\begin{array}{c}
\boldsymbol{\beta}_{k}^{V} \\
\boldsymbol{\alpha}_{k}^{V}
\end{array}\right]\right)=0 .
$$


In view of controllability, at least one of the errors is not zero and thus at least one of Eqs. (27) and (28) is satisfied, identifying the kernel.

In case both errors $\epsilon_{k+1}^{U}$ and $\epsilon_{k+1}^{V}$ are nonzero, we might have a choice between these representations of the kernel. In Theorem 1 we give explicitly which one should be selected, but in order to complete the recursive step, we will need to extend either $U_{k+1}^{m}$ or $V_{k+1}^{p}$ to make the kernel two dimensional and find an extra generating vector in this kernel. (We consider $j, r$ generic in the next lemma.) So, let $U_{k+1}^{j}$ and $V_{k+1}^{r}$ be partitioned as (25). Then

$$
\mathcal{A}_{k+1} U_{k+1}^{j}=\left[\begin{array}{cc}
\mathcal{A}_{k} & 0 \\
\boldsymbol{a}_{k+1} & \lambda_{k+1}
\end{array}\right]\left[\begin{array}{c}
U_{k}^{j} \\
u_{k+1}^{m}
\end{array}\right]=\left[\begin{array}{c}
\mathcal{A}_{k} U_{k}^{j} \\
\boldsymbol{a}_{k+1} U_{k}^{j}+\lambda_{k+1} u_{k+1}^{j}
\end{array}\right]
$$

and thus

$$
U_{k+1}^{j+1}=\left[U_{k+1}, \mathcal{A}_{k+1} U_{k+1}^{j}\right]=\left[\begin{array}{cc}
U_{k}, & \mathcal{A}_{k} U_{k}^{j} \\
u_{k+1}, & \boldsymbol{a}_{k+1} U_{k}^{j}+\lambda_{k+1} u_{k+1}^{j}
\end{array}\right]=\left[\begin{array}{c}
U_{k}^{j+1} \\
u_{k+1}^{j+1}
\end{array}\right] .
$$

A similar representation holds if we want to extend $V_{k+1}^{r}$ to $V_{k+1}^{r+1}$. We discuss now how to handle these extension and, crucially for defining the algorithm, which one to pick.

Lemma 5 Let $U_{k+1}^{j}, U_{k+1}^{j+1}$ be partitioned as (25) and let $\mathcal{A}_{k+1}$ be partitioned as in (20). Then, for any vector $\gamma$ of dimension $m$, it is

$$
u_{k+1}^{j+1}\left(\left[\begin{array}{l}
0 \\
\boldsymbol{\gamma}
\end{array}\right]-\lambda_{k+1}\left[\begin{array}{l}
\boldsymbol{\gamma} \\
0
\end{array}\right]\right)=\boldsymbol{a}_{k+1} U_{k}^{j} \boldsymbol{\gamma}
$$

Similarly,

$$
v_{k+1}^{r+1}\left(\left[\begin{array}{l}
0 \\
\boldsymbol{\gamma}
\end{array}\right]-\lambda_{k+1}\left[\begin{array}{l}
\boldsymbol{\gamma} \\
0
\end{array}\right]\right)=\boldsymbol{a}_{k+1} V_{k}^{r} \boldsymbol{\gamma}
$$

for $v_{k+1}^{r+1}$ in the partition equivalent to (25) of $V_{k+1}^{r+1}$.

Proof Notice that, in view of (29),

$$
u_{k+1}^{j+1}\left[\begin{array}{l}
0 \\
\boldsymbol{\gamma}
\end{array}\right]=\left(\boldsymbol{a}_{k+1} U_{k}^{j}+\lambda_{k+1} u_{k+1}^{j}\right) \boldsymbol{\gamma} \text {. }
$$

On the other hand, from (14), $U_{k+1}^{j+1}=\left[U_{k+1}^{j}, \mathcal{A}_{k+1}^{j+1} U_{k+1}\right]$ and thus

$$
u_{k+1}^{j+1} \lambda_{k+1}\left[\begin{array}{l}
\boldsymbol{\gamma} \\
0
\end{array}\right]=\lambda_{k+1} u_{k+1}^{j} \gamma
$$

Subtracting (33) from (32) yields the result. The corresponding equality for $v_{k+1}^{r+1}, V_{k+1}^{r+1}$ is proved similarly. 
Notice that the expressions in (30) and (31) do not depend on the last data points $u_{k+1}$ and $v_{k+1}$, respectively, a fact which is crucial to derive Eqs. (34) and (35) below.

We denote by $\overrightarrow{\boldsymbol{x}}=\left[0, x_{0}, x_{1}, \ldots, x_{k-1}\right]^{T}$ the downward shift of the vector $\boldsymbol{x}=$ $\left[x_{0}, x_{1}, x_{1}, \ldots, x_{k}\right]^{T}$. An immediate consequence of Lemma 5 is that, if $\left[\begin{array}{c}\boldsymbol{\beta}_{k}^{U} \\ \boldsymbol{\alpha}_{k}^{U}\end{array}\right]$ is in the kernel of $\left[U_{k}^{j},-V_{k}^{r}\right]$ and $\alpha_{k, r}^{U}=0$, then

$$
\left[U_{k+1}^{j+1},-V_{k+1}^{r}\right]\left(\left[\begin{array}{c}
0 \\
\boldsymbol{\beta}_{k}^{U} \\
\hline \overrightarrow{\boldsymbol{\alpha}}_{k}^{U}
\end{array}\right]-\lambda_{k+1}\left[\begin{array}{c}
\boldsymbol{\beta}_{k}^{U} \\
0 \\
\hline \boldsymbol{\alpha}_{k}^{U}
\end{array}\right]\right)=0 .
$$

Similarly, if $\left[\begin{array}{c}\boldsymbol{\beta}_{k}^{V} \\ \boldsymbol{\alpha}_{k}^{V}\end{array}\right]$ is in the kernel of $\left[U_{k}^{j},-V_{k}^{r}\right]$ and $\beta_{k, j}^{V}=0$, then

$$
\left[U_{k+1}^{j},-V_{k+1}^{r+1}\right]\left(\left[\begin{array}{c}
\overrightarrow{\boldsymbol{\beta}}_{k}^{V} \\
\hline 0 \\
\boldsymbol{\alpha}_{k}^{V}
\end{array}\right]-\lambda_{k+1}\left[\begin{array}{c}
\boldsymbol{\beta}_{k}^{V} \\
\boldsymbol{\alpha}_{k}^{V} \\
0
\end{array}\right]\right)=0 .
$$

We are now ready to construct an interpolant of $\left(\mathcal{A}_{k+1}, U_{k+1}, V_{k+1}\right)$. from a min-

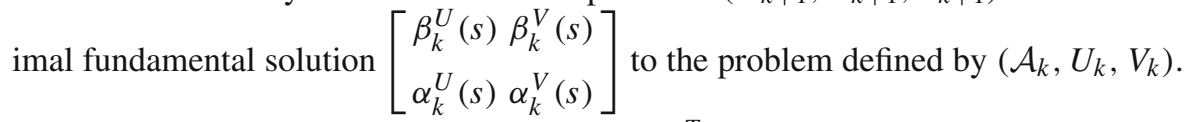
Notice that, if the vector $\boldsymbol{x}=\left[x_{0}, x_{1}, \ldots, x_{k}\right]^{T}$ represents the coefficients of the polynomial $x(s)$, then $\boldsymbol{x}^{\prime}:=\left[0, x_{0}, x_{1}, \ldots, x_{k}\right]^{T}$ represents the coefficients of $s x(s)$. Similarly, if the last entry $x_{k}$ of $\mathbf{x}$ vanishes, then also $\overrightarrow{\boldsymbol{x}}:=\left[0, x_{0}, x_{1}, \ldots, x_{k-1}\right]^{T}$ represents the coefficients of $s x(s)$.

Lemma 6 Let $\Gamma_{k}(s)=\left[\begin{array}{cc}\beta_{k}^{U} & \beta_{k}^{V} \\ \alpha_{k}^{U}, & \alpha_{k}^{V}\end{array}\right]$ with column degrees $\mu_{k}, v_{k}$ be a minimal fundamental solution to $\left(\mathcal{A}_{k}, U_{k}, V_{k}\right)$ nested in $\left(\mathcal{A}_{k+1}, U_{k+1}, V_{k+1}\right)$. Let $\left[\epsilon_{k+1}^{U}, \epsilon_{k+1}^{V}\right]$ be as in (26).

Then, if $\epsilon_{k+1}^{U} \neq 0$,

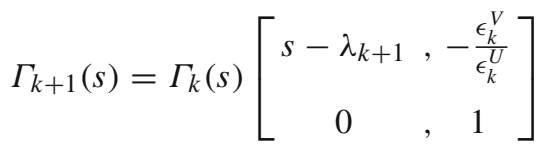


provides a fundamental solution to $\left(\mathcal{A}_{k+1}, U_{k+1}, V_{k+1}\right)$ column degrees $\mu_{k}+1, v_{k}$. Similarly, if $\epsilon_{k+1}^{V} \neq 0$,

$$
\Gamma_{k+1}(s)=\Gamma_{k}(s)\left[\begin{array}{cc}
1 & 0 \\
-\frac{\epsilon_{k}^{U}}{\epsilon_{k}^{V}} & , s-\lambda_{k+1}
\end{array}\right]
$$

provides a fundamental solution to $\left(\mathcal{A}_{k+1}, U_{k+1}, V_{k+1}\right)$ with column degrees $\mu_{k}, v_{k}+$ 1.

Proof Since $\Gamma_{k}(s)$ is a minimal fundamental solution, the degree of $\alpha_{k}^{U}(s)$ is smaller than $v_{k}$ and thus the vector representation of the first column in the identity (36) is

$$
\left[\begin{array}{c}
\boldsymbol{\beta}_{k+1}^{U} \\
\boldsymbol{\alpha}_{k+1}^{U}
\end{array}\right]=\left[\begin{array}{c}
0 \\
\boldsymbol{\beta}_{k}^{U} \\
\overrightarrow{\boldsymbol{\alpha}}_{k}^{U}
\end{array}\right]-\lambda_{k+1}\left[\begin{array}{c}
\boldsymbol{\beta}_{k}^{U} \\
0 \\
\hline \boldsymbol{\alpha}_{k}^{U}
\end{array}\right]
$$

which, in view of (34), is in the kernel of $\left[U_{k}^{\mu_{k}+1},-V_{k}^{v_{k}}\right]$, while the vector representation of the second column in the identity reads as

$$
\left[\begin{array}{c}
\boldsymbol{\beta}_{k+1}^{V} \\
\boldsymbol{\alpha}_{k+1}^{V}
\end{array}\right]=-\frac{\epsilon_{k+1}^{V}}{\epsilon_{k+1}^{U}}\left[\begin{array}{c}
\boldsymbol{\beta}_{k}^{U} \\
0 \\
\frac{\boldsymbol{\alpha}_{k}^{U}}{}
\end{array}\right]+\left[\begin{array}{c}
\boldsymbol{\beta}_{k}^{V} \\
0 \\
\frac{\boldsymbol{\alpha}_{k}^{V}}{}
\end{array}\right]
$$

which, in view of (27), is again easily seen to be in the kernel of the matrix $\left[U_{k}^{\mu_{k}+1},-V_{k}^{v_{k}}\right]$. Therefore $\Gamma_{k+1}(s)$ is an interpolant of for the problem defined by $\left(\mathcal{A}_{k+1}, U_{k+1}, V_{k+1}\right)$ with column degrees $\mu_{k}+1, v_{k}$.

Furthermore, it is a fundamental interpolant: in fact, if $\widehat{\Gamma}(s)$ is a minimal fundamental interpolant, there exists a polynomial matrix $P(s)$ such that $\widehat{\Gamma}(s) P(s)=\Gamma_{k+1}(s)$. Since the determinants of $\Gamma_{k+1}(s)$ (by construction) and $\widehat{\Gamma}(s)$ (by definition) are both $\chi_{\mathcal{A}_{k+1}}(s)\left(\chi_{\mathcal{A}}(s)\right.$ being the characteristic polynomial of $\left.\mathcal{A}\right)$, the matrix $P(s)$ is a unit. A similar reasoning holds for (37), using (28) and (35).

So, the recursive step appears to be quite simple. The problem is that we obtain a fundamental solution which is not minimal. Since the proof of Lemma 6 makes use of (34) and (35), we need the degree of $\alpha_{k}^{U}(s)$ to be less than $v_{k}$ or that of $\beta_{k}^{V}(s)$ to be less than $\mu_{k}$. There is a small modification of the above procedure which ensures that this condition is satisfied, making the recursion complete. Notice that if both $\epsilon_{k+1}^{U}$ and $\epsilon_{k+1}^{V}$ are different from zero then the cases $\mu_{k} \leq v_{k}$ and $\mu_{k}>v_{k}$ should be analyzed separately.

Theorem 1 Let $\left(\mathcal{A}_{k}, U_{k}, V_{k}\right)$ be a sequence of nested problems for $k \leq K$ and let $\Gamma_{k}(s)=\left[\begin{array}{cc}\beta_{k}^{U} & \beta_{k}^{V} \\ \alpha_{k}^{U}, & \alpha_{k}^{V}\end{array}\right]$ be as in (22) and such that (19) are satisfied and let $\left[\epsilon_{k+1}^{U}, \epsilon_{k+1}^{V}\right]$ be as in (26). 
Then, if $\epsilon_{k+1}^{U} \neq 0$ and either $\mu_{k} \leq v_{k}$ or $\epsilon_{k+1}^{V}=0$

$$
\Gamma_{k+1}(s)=\left[\begin{array}{cc}
\beta_{k+1}^{U} & \beta_{k+1}^{V} \\
\alpha_{k+1}^{U}, & \alpha_{k+1}^{V}
\end{array}\right]=\left[\begin{array}{cc}
\beta_{k}^{U} & \beta_{k}^{V} \\
\alpha_{k}^{U} & \alpha_{k}^{V}
\end{array}\right]\left[\begin{array}{cc}
s-\lambda_{k+1}-\frac{\epsilon_{k+1}^{V}\left[-\boldsymbol{\alpha}_{k}^{U}\right]_{v_{k}-1}}{\epsilon_{k+1}^{U}} & ,-\frac{\epsilon_{k+1}^{V}}{\epsilon_{k+1}^{U}} \\
{\left[-\boldsymbol{\alpha}_{k}^{U}\right]_{v_{k}-1}} & ,
\end{array}\right],
$$

where $[\boldsymbol{\alpha}]_{j}$ denotes the coefficient with index $j$ in the vector $\boldsymbol{\alpha}=\left[\alpha_{0}, \ldots, \alpha_{v_{k}}\right]^{T}$.

Similarly, if $\epsilon_{k+1}^{V} \neq 0$ and either $\mu_{k}>v_{k}$ or $\epsilon_{k+1}^{U}=0$

$$
\Gamma_{k+1}(s)=\left[\begin{array}{cc}
\beta_{k+1}^{U} & \beta_{k+1}^{V} \\
\alpha_{k+1}^{U}, & \alpha_{k+1}^{V}
\end{array}\right]=\left[\begin{array}{cc}
\beta_{k}^{U} & \beta_{k}^{V} \\
\alpha_{k}^{U} & \alpha_{k}^{V}
\end{array}\right]\left[\begin{array}{cc}
1 & , \\
\left.-\boldsymbol{\beta}_{k}^{V}\right]_{\mu_{k}-1} \\
-\frac{\epsilon_{k+1}^{U}}{\epsilon_{k+1}^{V}} & , s-\lambda_{k+1}-\frac{\epsilon_{k+1}^{U}\left[-\boldsymbol{\beta}_{k}^{V}\right]_{\mu_{k}-1}}{\epsilon_{k+1}^{V}}
\end{array}\right] .
$$

In both cases, $\Gamma_{k+1}(s)$ satisfies (19), that is it determines the minimal fundamental solution.

The proof will be given in Theorem 3, where the multivariable case is treated.

Let us point out that Eq. (38) implies that if $\epsilon_{k+1}^{U} \neq 0$ and $\mu_{k}<\nu_{k}$ then $\operatorname{deg} \alpha_{k}^{U}(s)<\operatorname{deg} \beta_{k}^{U}(s)<\operatorname{deg} \alpha_{k}^{V}(s)$ and therefore $\left[-\boldsymbol{\alpha}_{k}^{U}\right]_{v_{k}-1}=0$, which means that the polynomials $\beta_{k+1}^{U}$ and $\alpha_{k+1}^{U}$ are no longer coprime. Similarly, Eq. (39) implies that if $\epsilon_{k+1}^{V} \neq 0$ and $v_{k}<\mu_{k}-1$ then $\beta_{k+1}^{V}$ and $\alpha_{k+1}^{V}$ will have $\left(s-\lambda_{k+1}\right)$ as a common factor.

Notice that, in the second factor of (38) and (39), the determinant is always ( $s-$ $\left.\lambda_{k+1}\right)$, as expected.

\section{A general minimal degree solution for the tangential interpolation problem}

Let us return to the interpolation Problem 1 on matrix valued functions. Using the general notation $W=[U,-V]$ and, similarly to what was done in the scalar case, define, relatively to $(\mathcal{A}, W)$, where $W=\left[W_{1}, \ldots, W_{r}\right]$, the matrices $W_{i}^{\omega_{i}}$ for $i=$ $1, \ldots, r$ as

$$
W_{i}^{\omega_{i}}:=\left[W_{i}, \mathcal{A} W_{i}, \ldots, \mathcal{A}^{\omega_{i}} W_{i}\right] \text { for } i=1, \ldots, r
$$

and $\boldsymbol{W}^{\omega}:=\left[W_{1}^{\omega_{1}}, W_{2}^{\omega_{2}}, \ldots, W_{r}^{\omega_{r}}\right]$, where $\omega:=\left[\omega_{1}, \ldots, \omega_{r}\right]$. We set $\kappa^{i}$ to be the controllability index of $W_{i}$ in the pair $(\mathcal{A}, W)$, that is, the smallest exponent $\kappa^{i}$ such that

$$
\mathcal{A}^{\kappa^{i}} W_{i} \in \operatorname{span}\left\{W_{1}^{\kappa^{i}}, \ldots, W_{i-1}^{\kappa^{i}}, W_{i}^{\kappa^{i}-1}, W_{i+1}^{\kappa^{i}-1}, \ldots, W_{r}^{\kappa^{i}-1}\right\}
$$


Notice that this implies that the vectors in $\left[W_{1}^{\kappa^{1}-1}, W_{2}^{\kappa^{2}-1}, \ldots, W_{r}^{\kappa^{r}-1}\right]$ are linearly independent. On the other hand, in view of controllability, they span $\mathbb{C}^{K+1}$ and thus $\sum_{i=1}^{r} \kappa^{i}=K+1$. We denote by $\gamma_{i, j}(s)$ the $(i, j)$-th entry of $\Gamma(s)$ and set, for $j=1, \ldots r, \boldsymbol{\gamma}_{j}:=\left[\begin{array}{c}\boldsymbol{\gamma}_{1, j} \\ \boldsymbol{\gamma}_{2, j} \\ \vdots \\ \boldsymbol{\gamma}_{r, j}\end{array}\right]$, where $\boldsymbol{\gamma}_{i, j}$ denotes the vector formed by the coefficients $\left[\gamma_{i, j}\right]_{0},\left[\gamma_{i, j}\right]_{1}, \ldots\left[\gamma_{i, j}\right]_{\kappa^{i}}$ of the polynomial $\gamma_{i, j}(s)$ (we add zero coefficients of the higher powers if the actual degree of $\gamma_{i, j}(s)$ is lower than $\kappa^{i}$ ). That is

$$
\gamma_{i, j}(s)=\left[1, s, \ldots, s^{\kappa^{i}}\right] \boldsymbol{\gamma}_{i, j}=\sum_{k=0}^{\kappa^{i}}\left[\gamma_{i, j}\right]_{k} s^{k}
$$

We now have the analogous of Lemma 3, showing that a matrix polynomial $\gamma$ generates a solution to Problem 1 if and only if its coefficients determine linear dependencies among the columns of the controllability matrix $\left[W, \mathcal{A} W, \mathcal{A}^{2} W, \ldots\right]$ :

Theorem 2 Let $\gamma_{i, j}(s)=\sum_{k=0}^{\kappa^{i}}\left[\gamma_{i, j}\right]_{k} s^{k}$ for $i, j=1, \ldots, r$ be scalar valued polynomials.

Assume that for the integers $\omega_{1}, \ldots, \omega_{r}$ the inequalities $\omega_{i} \geq \operatorname{deg} \gamma_{i, j}(s), i=$ $1, \ldots, r$ hold.

Then condition (5) is equivalent to

$$
\boldsymbol{W}^{\omega} \boldsymbol{\gamma}_{j}=0 \text { for } j=1, \ldots, r
$$

with the corresponding

$$
\phi_{j}(s)=\sum_{i=1}^{r} \sum_{k=0}^{\omega_{i}}\left[\gamma_{i, j}\right]_{k} \sum_{l=0}^{k-1}\left(s^{l} \mathcal{A}^{k-l-1}\right) W_{i}
$$

in Eq. (5). Moreover, if $\gamma_{1, j}, \ldots, \gamma_{r, j}$ have a common factor $s-s_{0}$ that is

$$
\gamma_{i, j}(s)=\left(s-s_{0}\right) \widehat{\gamma}_{i, j}(s),
$$

$i=1, \ldots, r$, where $s_{0} \notin \sigma(\mathcal{A})$, then $\boldsymbol{W}^{\boldsymbol{\omega}-\boldsymbol{e}} \widehat{\boldsymbol{\gamma}}_{j}=0$, where all the components of the vector e are equal to 1 . 
Proof A straightforward computation gives that:

$$
\begin{aligned}
\boldsymbol{W}^{\omega} \boldsymbol{\gamma}_{j} & =\sum_{i=1}^{r} W_{i}^{\omega_{i}} \boldsymbol{\gamma}_{i, j} \\
& =\sum_{i=1}^{r} \sum_{k=0}^{\omega_{i}}\left[\gamma_{i, j}\right]_{k} \mathcal{A}^{k} W_{i} \\
& =\sum_{i=1}^{r} W_{i} \gamma_{i, j}(s)-\sum_{i=1}^{r} \sum_{k=0}^{\omega_{i}}\left[\gamma_{i, j}\right]_{k}\left(s^{k} I-\mathcal{A}^{k}\right) W_{i} \\
& =W \gamma_{j}(s)-(s I-\mathcal{A}) \sum_{i=1}^{r} \sum_{k=1}^{\omega_{i}}\left[\gamma_{i, j}\right]_{k} \sum_{l=0}^{k-1}\left(s^{l} \mathcal{A}^{k-l-1}\right) W_{i}
\end{aligned}
$$

Thus, setting $\phi_{j}(s)$ as in (42), we immediately get that (41) implies that $W \gamma_{j}(s)=$ $(s I-\mathcal{A}) \phi_{j}(s)$ for $j=1, \ldots r$, that is (5) holds.

Conversely, if for some matrix polynomial $\widetilde{\Phi(s)}$, Eq. (5) holds with $\Gamma(s)$, then for each column $\widetilde{\phi_{j}(s)}$, for $j=1, \ldots, r$, we have, substituting in (43), $\boldsymbol{W}^{\boldsymbol{p}} \boldsymbol{\gamma}^{i}=$ $(s I-\mathcal{A})\left(\phi_{j}(s)-\widetilde{\phi_{j}(s)}\right)$. The term on the left-hand side is constant, while the degree of the right-hand side - if it is not identically zero — is at least 1. Consequently, identity (41) should hold.

Suppose now $\gamma_{i, j}(s)=\left(s-s_{0}\right) \widehat{\gamma}_{i}, j(s), i=1, \ldots, r$, with $s_{0} \notin \sigma(\mathcal{A})$. Thus, setting $\left[\widehat{\gamma}_{i, j}\right]_{-1}=\left[\widehat{\gamma}_{i}, j\right]_{p_{i}}=0$ we can write $\left[\gamma_{i, j}\right]_{k}=\left[\widehat{\gamma}_{i, j}\right]_{k-1}-s_{0}\left[\widehat{\gamma}_{i}, j\right]_{k}$. Thus we have that:

$$
\begin{aligned}
\boldsymbol{W}^{\omega} \boldsymbol{\gamma}_{j} & =\sum_{i=1}^{r} W_{i}^{\omega_{i}} \boldsymbol{\gamma}_{i, j} \\
& =\sum_{i=1}^{r} \sum_{k=0}^{\omega_{i}}\left(\left[\widehat{\gamma}_{i, j}\right]_{k-1}-s_{0}\left[\widehat{\gamma}_{i, j}\right]_{k}\right) \mathcal{A}^{k} W_{i} \\
& =\sum_{i=1}^{r} \sum_{k=0}^{\omega_{i}}\left[\widehat{\gamma}_{i}, j\right]_{k-1} \mathcal{A}^{k} W_{i}-s_{0} \sum_{i=1}^{r} \sum_{k=0}^{\omega_{i}}\left[\widehat{\gamma}_{i, j}\right]_{k} \mathcal{A}^{k} W_{i} \\
& =\sum_{i=1}^{r} \sum_{k=0}^{\omega_{i}-1}\left[\widehat{\gamma}_{i, j}\right]_{k} \mathcal{A}^{k+1} W_{i}-s_{0} \sum_{i=1}^{r} \sum_{k=0}^{\omega_{i}-1}\left[\widehat{\gamma}_{i, j}\right]_{k} \mathcal{A}^{k} W_{i} \\
& =\left(\mathcal{A}-s_{0} I\right) \sum_{i=1}^{r} \sum_{k=0}^{\omega_{i}-1}\left[\widehat{\gamma}_{i, j}\right]_{k} \mathcal{A}^{k} W_{i} \\
& =\left(\mathcal{A}-s_{0} I\right) W^{\omega-e} \widehat{\gamma}_{j} .
\end{aligned}
$$

Since $\left(\mathcal{A}-s_{0} I\right)$ is invertible, we reach the desired conclusion.

Immediate consequence of the calculation in the previous Theorem is the following corollary. 
Corollary 1 Under the conditions of Theorem 2 the following representation holds

$$
\frac{1}{2 \pi i} \int_{\Delta}(s I-\mathcal{A})^{-1} W \gamma_{j}(s) d s=W^{\omega} \gamma_{j}
$$

where $\Delta$ is any simple closed curve with counterclockwise orientation around the eigenvalues of $\mathcal{A}$.

Proof Introducing the notation

$$
\phi_{j}(s)=\sum_{i=1}^{r} \sum_{k=1}^{\omega_{i}}\left[\gamma_{i, j}\right]_{k} \sum_{l=0}^{k-1}\left(s^{l} \mathcal{A}^{k-l-1}\right) W_{i}
$$

Equation (43) can be written in the form

$$
\boldsymbol{W}^{\omega} \boldsymbol{\gamma}_{j}=W \gamma_{j}(s)-(s I-\mathcal{A}) \phi_{j}(s)
$$

Multiplying both sides by $(s I-\mathcal{A})^{-1}$ and integrating, we obtain:

$$
\frac{1}{2 \pi i} \int_{\Delta}(s I-\mathcal{A})^{-1} W^{\omega} \gamma_{j} d s=\frac{1}{2 \pi i} \int_{\Delta}(s I-\mathcal{A})^{-1} W \gamma_{j}(s) d s .
$$

Using that $\frac{1}{2 \pi i} \int_{\Delta}(s I-\mathcal{A})^{-1} d s=I$ we get (44).

As we have pointed out after Eq. (5) the polynomial matrix $\gamma$ generates a solution to Problem 1 if together with $\phi(s)=(s I-\mathcal{A})^{-1} W \gamma(s)$ form a polynomial inputoutput pair. According to Theorem 5 in Forney [7] the column degrees of a minimal polynomial basis of the polynomial input-output pairs coincide with the controllability indexes of the pair $(\mathcal{A}, W)$. This connection leads to the following definition.

Definition 3 We say that $\Gamma(s)=\left[\gamma_{1}(s), \ldots, \gamma_{r}(s)\right]$ is a minimal fundamental solution (MF solution) to the interpolation Problem 1 determined by $(\mathcal{A}, W)$ if there exists a matrix polynomial $\Phi$ such that (4) is satisfied and, for each column $\gamma_{j}(s)=$ $\left[\begin{array}{c}\gamma_{1, j}(s) \\ \gamma_{2, j}(s) \\ \vdots \\ \gamma_{r, j}(s)\end{array}\right]$ of $\Gamma(s)($ for $j=1, \ldots, r)$,

$$
\begin{aligned}
& \operatorname{deg} \gamma_{j, j}(s)=\kappa^{j}, \quad \gamma_{j, j}(s) \text { is monic } \\
& \operatorname{deg} \gamma_{i, j}(s) \leq \min \left(\kappa^{i}-1, \kappa^{j}\right), \quad \text { for } 1 \leq i, j \leq r, i \neq j
\end{aligned}
$$

where $\kappa^{j}, j=1, \ldots r$ are the controllability indexes of $(\mathcal{A}, W)$.

Remark 6 Let us observe that an immediate consequence of the degree constraints in the MF solution that it will be column reduced. This property plays a crucial role in the analysis of the minimal possible McMillan degree of the interpolants in [3]. 
The following proposition shows that the MF solution is essentially equivalent to a basis selection scheme corresponding to the controllability indexes of the pair $(\mathcal{A}, W)$ (Cf. Section 6.7.2 in [12]). This statement follows from the more general analysis presented in [2] which is based on the so-called nice selections defined by Youngdiagrams, but we present here a short proof of it which fits more to our purposes.

Proposition 4 For any controllable set of data $(\mathcal{A}, W)$ there exists a unique MF solution.

Proof By construction, the set

$$
\left\{W_{1}, \mathcal{A} W_{1}, \ldots, \mathcal{A}^{\kappa^{1}-1} W_{1}, W_{2}, \mathcal{A} W_{2}, \ldots, \mathcal{A}^{\kappa^{r}-1} W_{r}\right\}
$$

constitutes a basis for $\mathbb{C}^{K+1}$. This basis is uniquely determined by the order of the columns of $W$. Thus, for $j=1, \ldots, r$, there exist unique vectors $\hat{\gamma}_{1, j}, \ldots, \hat{\gamma}_{r, j}$ of dimensions $\kappa^{1}, \ldots, \kappa^{r}$, such that

$$
\mathcal{A}^{\kappa^{j}} W_{j}+\sum_{i=1}^{r}\left[W_{i}, \mathcal{A} W_{i}, \ldots, \mathcal{A}^{\kappa^{i}-1} W_{i}\right] \hat{\boldsymbol{\gamma}}_{i, j}=0
$$

Set now, for $j=1, \ldots, r$,

$$
\boldsymbol{\gamma}_{j, j}:=\left[\begin{array}{c}
\hat{\boldsymbol{\gamma}}_{j, j} \\
1
\end{array}\right] \quad \boldsymbol{\gamma}_{i, j}:=\left[\begin{array}{c}
\hat{\boldsymbol{\gamma}}_{i, j} \\
0
\end{array}\right] \text { for } j \neq i .
$$

Then (47) can be rewritten as:

$$
\sum_{i=1}^{r}\left[W_{i}, \mathcal{A} W_{i}, \ldots, \mathcal{A}^{\kappa^{i}} W_{i}\right] \boldsymbol{\gamma}_{i, j}=0
$$

Therefore, in view of Theorem (2), for each $j=1, \ldots, r$, the vector-polynomial $\gamma_{j}(s)$ corresponding to $\boldsymbol{\gamma}_{1, j} \ldots, \boldsymbol{\gamma}_{r, j}$ as in (40) provides a solution to Problem 1.

Furthermore, the polynomials $\gamma_{1, j}(s), \gamma_{2, j}(s), \ldots, \gamma_{r, j}(s)$ associated with the vectors $\gamma_{j}$ will have degree at most $\kappa^{i}-1$ if $i \neq j$ and $\kappa^{i}$ if $i=j$. On the other hand, by construction, for $j=1, \ldots, r$ only the components of (46) with exponent $l \leq \kappa^{j}$ are needed, which means that $\operatorname{deg} \boldsymbol{\gamma}_{i, j}(s) \leq \kappa^{j}$. Thus, the following inequalities hold:

$$
\operatorname{deg} \gamma_{i, j}(s) \begin{cases}\leq \min \left(\kappa^{i}-1, \kappa^{j}\right) & \text { if } i \neq j \\ =\kappa^{j} & \text { if } i=j\end{cases}
$$

and $\gamma_{j, j}(s)$ will be monic. Thus conditions (45) are satisfied. Moreover, for each column $j$ the degree of its $j$-th entry is exactly $\kappa^{j}$ and it is not greater than $\kappa^{j}$ if $i<j$ and strictly less than $j$ if $i>j$. Thus, setting $\Gamma(s):=\left[\gamma_{1}(s), \gamma_{2}(s), \ldots, \gamma_{r}(s)\right]$, its column highest degree coefficient matrix is a triangular matrix with the diagonal equal 
to the identity. Therefore $\operatorname{deg} \operatorname{det} \Gamma(s)=\sum_{j} \kappa^{j}=K+1$. In particular, $\operatorname{det} \Gamma(s)$ is not identically zero. (Moreover, $\Gamma$ is column reduced.)

To prove uniqueness, observe that, if $\bar{\Gamma}(s)$ is another solution to (4) which satisfies (45), then the difference $\tilde{\Gamma}(s):=\Gamma(s)-\bar{\Gamma}(s)$ is such that, for each $j=1, \ldots r$, $\operatorname{deg} \tilde{\gamma}_{j, j}(s)<\kappa^{j}$. Thus, in view of (45), it is

$$
\begin{aligned}
\max _{i: \kappa_{i} \leq \kappa_{j}} \operatorname{deg} \tilde{\gamma}_{i, j}(s) & \leq \min \left(\left(\kappa_{j}-1\right), \max _{i: i \neq j, \kappa_{i} \leq \kappa_{j}}\left(\kappa_{i}-1\right)\right) \\
& =\kappa_{j}-1<\operatorname{deg} \gamma_{j, j}(s) \quad j=1, \ldots, r .
\end{aligned}
$$

In particular

$$
\operatorname{deg} \tilde{\gamma}_{j}(s)<\operatorname{deg} \gamma_{j}(s)
$$

On the other hand, $\tilde{\Gamma}(s)$ still satisfies (4) and thus each of its columns $\tilde{\gamma}_{j}(s)$ can be expressed in terms of the columns of the fundamental solution $\Gamma(s)$ : thus there exists a polynomial $\tilde{\pi}_{j}(s)$ such that $\tilde{\gamma}_{j}(s)=\Gamma(s) \tilde{\pi}_{j}(s)$. Since $\Gamma(s)$ is column reduced, it has the predictable degree property (see [12, Theorem 6.3-13], that is,

$$
\operatorname{deg} \tilde{\gamma}_{j}(s)=\max _{l ; \tilde{\pi}_{l, j}(s) \neq 0}\left\{\operatorname{deg} \gamma_{l}(s)+\operatorname{deg} \tilde{\pi}_{l, j}\right\}
$$

where $\tilde{\pi}_{l, j}(s)$ is the $l$-th row of $\tilde{\pi}_{j}(s)$ for $l=1, \ldots r$.

Let us introduce the notation $\tau_{1}=\max _{j} \kappa_{j}$. From Eqs. (50) and (51) it is immediate that $\tilde{\pi}_{i, j}=0$, if $\kappa_{i}=\tau_{1}$.

Consequently, the following representation holds:

$$
\tilde{\gamma}_{i, j}=\sum_{l: \kappa_{l}<\tau_{1}} \gamma_{i, l} \tilde{\pi}_{l, j} .
$$

Let us observe that rearranging the columns (and the rows accordingly) of $\Gamma$ according to the decreasing order of the controllability indexes the highest column degree coefficients matrix is transformed into an upper triangular matrix with diagonal entries equal to 1 ; thus, any of its principal submatrix is of full rank, and thus, the corresponding part of the matrix $\Gamma$ has the predictable degree property. Reduce now the column vectors of $\tilde{\Gamma}$ keeping only those entries $\tilde{\gamma}_{i, j}$ for which $\kappa_{i}<\tau_{1}$. Now, let us introduce the notation: $\tau_{2}=\max \left\{\kappa_{i} \mid \kappa_{i}<\tau_{1}\right\}$. Since the submatrix of $\Gamma$ formed by the entries $\gamma_{i, l}$ for which $\kappa_{i} \leq \tau_{2}$ and $\kappa_{l} \leq \tau_{2}$ also has the degree predictable property we have that

$$
\max _{i: \kappa_{i} \leq \tau_{2}} \operatorname{deg} \tilde{\gamma}_{i, j}=\max _{l: \kappa_{l} \leq \tau_{2}, \pi_{l, j} \neq 0}\left(\operatorname{deg} \gamma_{l}+\operatorname{deg} \tilde{\pi}_{l, j}\right)
$$

Using again inequality (49) we get that $\max _{i: \kappa_{i} \leq \tau_{2}} \operatorname{deg} \tilde{\gamma}_{i, j}<\tau_{2}$, consequently $\tilde{\pi}_{l, j}=$ 0 , if $\kappa_{l}=\tau_{2}$, for $j=1, \ldots, r$. 
The argument can be repeated for the matrix obtained from $\tilde{\Gamma}$ after eliminating those entries $\tilde{\gamma}_{i, j}$ for which $\kappa_{i}<\tau_{2}$, and so on, finally proving that $\tilde{\pi}_{l, j}(s)=0$ for $l, j=1, \ldots, r$, thus $\tilde{\Gamma}(s)=0$, achieving the proof.

Remark 7 The uniqueness of the solution $\Gamma(s)$ hinges on both of the conditions in (45) and thus it relies on the controllability indexes of $(\mathcal{A}, W)$. If $\Gamma^{\prime}(s)$ is another column-reduced matrix polynomial satisfying only the second condition in (45) then using Theorem 6.5-4. in [12] we see that $\Gamma^{\prime}(s)$ can be obtained from $\Gamma(s)$ multiplying it from the right by a unimodular matrix; consequently, Lemma 6.3-14. in [12] gives us that the column degrees of $\Gamma(s)$ and $\Gamma^{\prime}(s)$-after an appropriate permutation of the columns-coincide.

\section{General multivariable recursive interpolation}

Similarly to the scalar case, given a controllable pair $(\mathcal{A}, W)$, with $\mathcal{A}$ lower triangular of dimension $(K+1) \times(K+1)$ and $W:=\left[W_{1}, W_{2}, \ldots, W_{r}\right]$ of dimension $(K+1) \times r$, we can consider a sequence of reduced interpolation problems $\left(\mathcal{A}_{k}, W_{k}\right)$ where $\mathcal{A}_{k}$ is the submatrix of the first $k+1$ rows and columns of $\mathcal{A}$ and $W_{k}=\left[W_{1, k}, \ldots, W_{r, k}\right]$ the truncation of $W$ to its first $k+1$ rows. According to Lemma 2 this means in particular to find right coprime polynomial matrices $\Phi_{k}(s), \Gamma_{k}(s)$ such that

$$
\left(s I-\mathcal{A}_{k}\right) \Phi_{k}=W_{k} \Gamma_{k}(s)
$$

Again, we can define $W_{j, k}^{\omega}:=\left[W_{j, k}, \mathcal{A}_{k} W_{j, k}, \ldots, \mathcal{A}_{k}^{\omega} W_{j, k}\right]$. It is immediate to see that

$$
W_{j, k}^{\omega+1}=\left[W_{j, k}, \mathcal{A}_{k} W_{j, k}^{\omega}\right]=\left[W_{j, k}^{p}, \mathcal{A}_{k}^{\omega+1} W_{j, k}\right]
$$

so that, if $\left[x_{0}, \ldots, x_{\omega}\right]^{T} \in \operatorname{ker} W_{j, k}^{\omega}$, both $\left[x_{0}, \ldots, x_{\omega}, 0\right]^{T}$ and $\left[0, x_{0}, \ldots, x_{\omega}\right]^{T}$ are in the kernel of $W_{j, k}^{\omega+1}$.

We can thus consider the sequence of nested (interpolation) problems $\left(\mathcal{A}_{k}, W_{k}\right)$ where, for each $k$,

$$
\mathcal{A}_{k+1}=\left[\begin{array}{cc}
\mathcal{A}_{k} & 0 \\
\boldsymbol{a}_{k+1} & \lambda_{k+1}
\end{array}\right] \quad W_{k+1}=\left[\begin{array}{c}
W_{k} \\
w_{k+1}
\end{array}\right]
$$

and set

$$
\boldsymbol{W}_{k}^{\kappa_{k}}:=\left[W_{1, k}^{\kappa_{k}^{1}}, W_{2, k}^{\kappa_{k}^{2}}, \ldots, W_{r, k}^{\kappa_{k}^{r}}\right]
$$

where $W_{k, i}^{\kappa_{k}^{i}}=\left[W_{j, k}, \mathcal{A}_{k} W_{j, k}, \ldots, \mathcal{A}_{k}^{\kappa_{k}^{i}} W_{j, k}\right]$, the index $\kappa_{k}^{i}$ is the controllability index of $W_{j, k}$ in $\left(\mathcal{A}_{k}, W_{k}\right)$ and $\kappa_{k}:=\left[\kappa_{k}^{1}, \ldots, \kappa_{k}^{r}\right]$ as a row vector. Let us point out that the lower triangular assumption on $\mathcal{A}$ implies that the vector formed from the first $k$ 
coordinates of the vector $\mathcal{A}_{k+1}^{m} W_{j, k+1}$ is equal to the vector $\mathcal{A}_{k}^{m} W_{j, k}$ for any $m \geq 0$, $j=1, \ldots, r, k \geq 1$, and consequently $\kappa_{k}^{j} \leq \kappa_{k+1}^{j}$ for all $j=1, \ldots, r, k \geq 1$.

(Let us point out that we assume $\left(\mathcal{A}_{k}, W_{k}\right)$ controllable for each $k$. Now since $\mathcal{A}$ is lower triangular, this can be achieved if for example the first entry of a column of $W$ and at least one element of each row of $\mathcal{A}$ before the diagonal are not zero. Another possibility is given when the diagonal elements of $\mathcal{A}$ are different and nonzero, and in each row of $W$ there is a nonzero element.)

Comparing the previous inequalities to the identities $\sum_{j=1}^{r} \kappa_{k}^{j}=k+1$, $\sum_{j=1}^{r} \kappa_{k+1}^{j}=k+2$ we obtain that there is only one controllability index which changes during the step $k \rightarrow k+1$. To locate this let us introduce the following notation:

For $k, j$ fixed and $i=1, \ldots, r$ let us denote by $\gamma_{i, j}^{k}$ a column vector of dimension $\kappa_{k}^{i}+1$ defined by the coefficients of the polynomial $\gamma_{i, j}^{k}(s)$ (which is the $(i, j)^{\text {th }}$ entry of $\Gamma_{k}(s)$ - —extended by extra zeros if necessary — and we set

$$
\boldsymbol{\gamma}_{j}^{k}:=\left[\begin{array}{c}
\boldsymbol{\gamma}_{1, j}^{k} \\
\boldsymbol{\gamma}_{2, j}^{k} \\
\vdots \\
\boldsymbol{\gamma}_{r, j}^{k}
\end{array}\right]
$$

Notice that

$$
\boldsymbol{W}_{k}^{\boldsymbol{\kappa}_{k}} \boldsymbol{\gamma}_{j}^{k}=0 \text { for } j=1, \ldots, r
$$

(see (48)).

Furthermore, let $\boldsymbol{w}_{k+1}^{\kappa_{k}}:=\left[w_{k+1}^{\kappa_{1}}, w_{k+1}^{\kappa_{2}}, \ldots, w_{k+1}^{\kappa_{r}}\right]$ denote the last row of the extended matrix

$$
\boldsymbol{W}_{k+1}^{\kappa_{k}}:=\left[W_{1, k+1}^{\kappa_{k}^{1}}, W_{2, k+1}^{\kappa_{k}^{2}}, \ldots, W_{r, k+1}^{\kappa_{k}^{r}}\right]
$$

where $W_{j, k+1}^{\kappa_{k}^{j}}=\left[W_{j, k+1}, \mathcal{A}_{k+1} W_{j, k+1}, \ldots, \mathcal{A}_{k+1}^{\kappa_{k}^{i}} W_{j, k+1}\right]$. Let us observe that due to the assumption that $\mathcal{A}$ is lower triangular we have that

$$
\boldsymbol{W}_{k+1}^{\boldsymbol{\kappa}_{k}}=\left[\begin{array}{c}
\boldsymbol{W}_{k}^{\boldsymbol{\kappa}_{k}} \\
\boldsymbol{w}_{k+1}^{\boldsymbol{k}_{k}}
\end{array}\right] \quad W_{j, k+1}^{\kappa_{k}^{j}}=\left[\begin{array}{c}
W_{j, k}^{\kappa_{k}^{j}} \\
w_{j, k+1}^{\kappa_{k}^{j}}
\end{array}\right] j=1, \ldots r
$$

and thus, similarly to the scalar case,

$$
\mathcal{A}_{k+1} W_{j, k+1}^{\kappa_{k}^{j}}=\left[\begin{array}{cc}
\mathcal{A}_{k} & 0 \\
\boldsymbol{a}_{k+1} & \lambda_{k+1}
\end{array}\right]\left[\begin{array}{c}
W_{j, k}^{\kappa_{k}^{j}} \\
w_{j, k+1}^{\kappa_{k}^{j}}
\end{array}\right]=\left[\begin{array}{c}
\mathcal{A}_{k} W_{j, k}^{\kappa_{k}^{j}} \\
\boldsymbol{a}_{k+1} W_{j, k}^{\kappa_{k}^{j}}+\lambda_{k+1} w_{j, k+1}^{\kappa_{k}^{j}}
\end{array}\right]
$$


and eventually, for $j=1, \ldots, r$,

$$
W_{j, k+1}^{\kappa_{k}^{j}+1}=\left[W_{j, k+1}, \mathcal{A}_{k+1} W_{j, k+1}^{\kappa_{k}^{j}}\right]=\left[\begin{array}{cc}
W_{j, k} & , \\
w_{j, k+1} & \mathcal{A}_{k} W_{j, k}^{\kappa_{k}^{j}} \\
\boldsymbol{a}_{k+1} W_{j, k}^{\kappa_{k}^{j}}+\lambda_{k+1} w_{j, k+1}^{\kappa_{k}^{j}}
\end{array}\right] .
$$

Finally, set

$$
\boldsymbol{\epsilon}_{k+1}=\left[\epsilon_{k+1}^{1}, \epsilon_{k+1}^{2}, \ldots, \epsilon_{k+1}^{r}\right]:=\boldsymbol{w}_{k+1}^{\kappa_{k}}\left[\boldsymbol{\gamma}_{1}^{k}, \boldsymbol{\gamma}_{2}^{k}, \ldots, \boldsymbol{\gamma}_{r}^{k}\right] .
$$

Let us observe that if $\epsilon_{k+1}^{j}=0$ for some $j$, then $\kappa_{k+1}^{j}=\kappa_{k}^{j}$. (The same linear combination which "worked" for the first $k$ coordinates also gives zero at the last position.) Notice as well that, since $\boldsymbol{W}_{k+1}^{k_{k}}$ has $k+r+1$ columns and $k+2$ rows, its kernel has dimension $r-1$, in view of controllability. Thus $\epsilon_{k+1} \neq 0$, since $\boldsymbol{W}_{k}^{\kappa k}\left[\boldsymbol{\gamma}_{1}^{k}, \boldsymbol{\gamma}_{2}^{k}, \ldots, \boldsymbol{\gamma}_{r}^{k}\right]=0$ and $\left[\boldsymbol{\gamma}_{1}^{k}, \boldsymbol{\gamma}_{2}^{k}, \ldots, \boldsymbol{\gamma}_{r}^{k}\right]$ is of full rank.

The following lemma shows how to locate which controllability index changes during the step $k \rightarrow k+1$.

Lemma 7 Suppose that $\left(\mathcal{A}_{k}, W_{k}\right)$ has controllability indexes $\kappa_{k}^{1}, \ldots, \kappa_{k}^{r}$ and $\left(\mathcal{A}_{k+1}, W_{k+1}\right)$ are as in (55). Let us order the set of controllability indexes $\kappa_{k}^{l}$, $l=1, \ldots, r$ according to their value and in case some of them are equal then according to the index $l$. Let $j$ be such that $\kappa_{k}^{j}$ is the smallest index (in this ordering) for which $\epsilon_{k+1}^{j} \neq 0$.

Then $\kappa_{k+1}^{j}=\kappa_{k}^{j}+1$.

Proof For any $j \in\{1, \ldots, r\}$ such that $\epsilon_{k+1}^{j} \neq 0$, setting

$$
\hat{\boldsymbol{\gamma}}_{i}^{k}:=\boldsymbol{\gamma}_{i}^{k}-\frac{\epsilon_{k+1}^{i}}{\epsilon_{k+1}^{j}} \boldsymbol{\gamma}_{j}^{k}
$$

we obtain

$$
\boldsymbol{W}_{k}^{\boldsymbol{\kappa}_{k}} \hat{\boldsymbol{\gamma}}_{i}^{k}=0 \quad i \neq j
$$

in view of (56), (57) and (59). We claim that the controllability indexes of $\left(\mathcal{A}_{k+1}, W_{k+1}\right)$ satisfy $\kappa_{k+1}^{i}=\kappa_{k}^{i}$ for $i \neq j$. The claim is immediate if $\epsilon_{k+1}^{i}=0$. Otherwise, we can write (61) as

$$
\boldsymbol{W}_{k}^{\kappa_{k}} \hat{\boldsymbol{\gamma}}_{k}^{i}=\sum_{l=1}^{r} W_{l, k+1}^{\kappa_{k}^{l}}\left(\boldsymbol{\gamma}_{l, k}^{i}-\frac{\epsilon_{k+1}^{i}}{\epsilon_{k+1}^{j}} \boldsymbol{\gamma}_{l, k}^{j}\right) \quad i \neq j
$$

where the highest nonzero component in each $\hat{\gamma}_{l, k}^{i}$ should be the $\left(\kappa_{k}^{i}+1\right)-\mathrm{st}$ if $l \leq i$ and $\left(\kappa_{k}^{i}\right)$-th if $l>i$ (both $\boldsymbol{\gamma}_{k}^{i}$ and $\boldsymbol{\gamma}_{k}^{j}$ have this property). If $\kappa_{k}^{i}>\kappa_{k}^{j}$ the condition 
is automatically satisfied; therefore $\kappa_{k+1}^{i}=\kappa_{k}^{i}$. This is always the case if $i<j$. If $\kappa_{k}^{i}=\kappa_{k}^{j}$, then the highest nonzero component of $\gamma_{l, k}^{i}$ is the $\left(\kappa_{k}^{i}\right)$-th for $l>i$, whereas the highest nonzero component of $\boldsymbol{\gamma}_{l, k}^{j}$ is the $\left(\kappa_{k}^{j}\right)-$ th for $l>j$. In view of the ordering, it is $i>j$ and, therefore, the condition is satisfied also for $\hat{\boldsymbol{\gamma}}_{k}^{i}$, proving the claim.

Since, in view of controllability, the sum of the controllability indexes has to increase by 1 , the only possibility is $\kappa_{k+1}^{j}=\kappa_{k}^{j}+1$. Thus, the controllability indexes of $\left(\mathcal{A}_{k+1}, W_{k+1}\right)$ are $\kappa_{k}^{1}, \ldots, \kappa_{k}^{j-1}, \kappa_{k}^{j}+1, \kappa_{k}^{j+1}, \ldots, \kappa_{k}^{r}$.

In the proof of the recursive scheme for the scalar case Lemma 6 played an important role which was based onto Lemma 5. The multivariate version of this latter one is formulated below.

Lemma 8 Let $W_{j, k+1}^{\kappa_{k}^{j}}$ be partitioned as (57) and let $\mathcal{A}_{k+1}$ be partitioned as in (55). Then, for any vector $\gamma$ of dimension $\kappa_{k}^{j}+1$, we have that

$$
w_{j, k+1}^{\kappa_{k}^{j}+1}\left(\left[\begin{array}{l}
0 \\
\boldsymbol{\gamma}
\end{array}\right]-\lambda_{k+1}\left[\begin{array}{l}
\boldsymbol{\gamma} \\
0
\end{array}\right]\right)=\boldsymbol{a}_{k+1} W_{j, k}^{\kappa_{k}^{j}} \boldsymbol{\gamma} .
$$

The proof is similar to that of the scalar case in Lemma 5 and it will not be repeated here.

Again-using the observation that the last entry of the vector $\boldsymbol{\gamma}_{i, j}^{k}$ is zero for $i \neq j$, an immediate consequence of Lemma 8 is that, if $\boldsymbol{\gamma}_{j}^{k} \in \operatorname{ker} \boldsymbol{W}_{k, i}^{\boldsymbol{K}_{k}}$, then

$$
\left[\begin{array}{c}
\overrightarrow{\boldsymbol{\gamma}}_{1, j}^{k} \\
\vdots \\
\overrightarrow{\boldsymbol{\gamma}}_{j-1, j}^{k} \\
\hline 0 \\
\frac{\boldsymbol{\gamma}_{j, j}^{k}}{\overrightarrow{\boldsymbol{\gamma}}_{j+1, j}^{k}} \\
\vdots \\
\overrightarrow{\boldsymbol{\gamma}}_{r, j}^{k}
\end{array}\right]-\lambda_{k+1}\left[\begin{array}{c}
\boldsymbol{\gamma}_{1, j}^{k} \\
\vdots \\
\frac{\boldsymbol{\gamma}_{j-1, j,}^{k}}{\boldsymbol{\gamma}_{j, j}^{k}} \\
\frac{0}{\boldsymbol{\gamma}_{j+1, j}^{k}} \\
\vdots \\
\boldsymbol{\gamma}_{r, j}^{k}
\end{array}\right] \in \operatorname{ker} \boldsymbol{W}_{j, k+1}^{\boldsymbol{\kappa}_{k}+e_{j}^{T}},
$$

where $e_{j}$ denotes the $j^{\text {th }} r$-dimensional unit vector.

Set furthermore

$$
\rho_{i, j}^{k}:= \begin{cases}0 & \text { for } i=j \\ {\left[\boldsymbol{\gamma}_{i, j}^{k}\right]_{\kappa_{k}^{i}-1}} & \text { for } i \neq j\end{cases}
$$

and $\boldsymbol{\rho}_{j}^{k}:=\left[\rho_{1, j}^{k}, \ldots \rho_{r, j}^{k}\right]$, where, we recall, $\left[\boldsymbol{\gamma}_{i, j}^{k}\right]_{\kappa_{k}^{i}-1}$ denotes the entry with index $\kappa_{k}^{i}-1$ of the $\kappa_{i}+1$-dimensional vector $\left[\left[\boldsymbol{\gamma}_{i, j}^{k}\right]_{0},\left[\boldsymbol{\gamma}_{i, j}^{k}\right]_{1}, \ldots,\left[\boldsymbol{\gamma}_{i, j}^{k}\right]_{\kappa_{k}^{i}-1},\left[\boldsymbol{\gamma}_{i, j}^{k}\right]_{\kappa_{k}^{i}},\right]^{T}$. Notice that, in view of (45), $\rho_{i, j}^{k}=0$ if $\kappa_{j}<\kappa_{i}-1$. 
Theorem 3 Let $\left(\mathcal{A}_{k}, W_{k}\right)$ be controllable pairs with $\mathcal{A}_{k}$ lower triangular for $k \leq K$ and let $\Gamma_{k}(s)=\left[\gamma_{1}^{k}(s), \ldots, \gamma_{r}^{k}(s)\right]$ be as in (53) (for some $\left.\Phi_{k}\right)$ and such that (45) are satisfied (that is, $\Gamma_{k}$ is a MF solution). Define $\epsilon_{k+1}^{l}$ as in (59) for $l=1, \ldots, r$ and let $j$ denote the index for which $\kappa_{k+1}^{j}=\kappa_{k}^{j}+1$.

Then $\Gamma_{k+1}(s)=\left[\gamma_{1}^{k+1}(s), \ldots, \gamma_{r}^{k+1}(s)\right]$ defined as

$$
\gamma_{i}^{k+1}(s)=\gamma_{i}^{k}(s)-\frac{\epsilon_{k+1}^{i}}{\epsilon_{k+1}^{j}} \gamma_{j}^{k}(s), \quad i \neq j
$$

and

$$
\gamma_{j}^{k+1}(s)=\left(s-\lambda_{k+1}\right) \gamma_{j}^{k}(s)-\sum_{l=0}^{r} \rho_{l, j}^{k} \gamma_{l}^{k+1}(s)
$$

is an $M F$ solution for $\left(\mathcal{A}_{k+1}, W_{k+1}\right)$.

Proof Due to the definition of the index $j$ to find the matrix polynomial $\Gamma_{k+1}(s)$ satisfying (45) we have to consider special elements in the kernel of $\left[W_{1, k+1}^{\kappa_{k}^{1}}, W_{2, k+1}^{\kappa_{k}^{2}} \ldots\right.$, $\left.W_{j, k+1}^{\kappa_{k}^{j}+1}, \ldots, W_{r, k+1}^{\kappa_{k}^{r}}\right]$

To this aim, for $i \neq j$, set

$$
\boldsymbol{\gamma}_{i}^{k+1}:=\left[\begin{array}{c}
\boldsymbol{\gamma}_{1, k}^{i} \\
\vdots \\
\frac{\boldsymbol{\gamma}_{j-1, i}^{k}}{\boldsymbol{\gamma}_{j, i}^{k}} \\
\frac{0}{\boldsymbol{\gamma}_{j+1, i}^{k}} \\
\vdots \\
\boldsymbol{\gamma}_{r, i}^{k}
\end{array}\right]-\frac{\epsilon_{k+1}^{i}}{\epsilon_{k+1}^{j}}\left[\begin{array}{c}
\boldsymbol{\gamma}_{1, j}^{k} \\
\vdots \\
\frac{\boldsymbol{\gamma}_{j-1, j}^{k}}{\boldsymbol{\gamma}_{j, j}^{k}} \\
\frac{0}{\boldsymbol{\gamma}_{j+1, j}^{k}} \\
\vdots \\
\boldsymbol{\gamma}_{r, j}^{k}
\end{array}\right]
$$

and, for $i=j$,

$$
\boldsymbol{\gamma}_{j}^{k+1}:=\left[\begin{array}{c}
\overrightarrow{\boldsymbol{\gamma}}_{1, j}^{k} \\
\vdots \\
\overrightarrow{\boldsymbol{\gamma}}_{j-1, j}^{k} \\
0 \\
\frac{\boldsymbol{\gamma}_{j, j}^{k}}{\overrightarrow{\boldsymbol{\gamma}}_{j+1, j}^{k}} \\
\vdots \\
\overrightarrow{\boldsymbol{\gamma}}_{r, j}^{k}
\end{array}\right]-\lambda_{k+1}\left[\begin{array}{c}
\boldsymbol{\gamma}_{1, j}^{k} \\
\vdots \\
\frac{\boldsymbol{\gamma}_{j-1, j}^{k}}{\boldsymbol{\gamma}_{j, j}^{k}} \\
\frac{0}{\boldsymbol{\gamma}_{j+1, j}^{k}} \\
\vdots \\
\boldsymbol{\gamma}_{r, j}^{k}
\end{array}\right]-\sum_{l=0}^{r} \rho_{l, j}^{k} \boldsymbol{\gamma}_{l}^{k+1}
$$


Clearly, in view of (54), (66) and (67) we have

$$
\left[W_{1, k+1}^{\kappa_{1}}, W_{2, k+1}^{\kappa_{2}} \ldots, W_{j, k+1}^{\kappa_{j}+1}, \ldots, W_{r, k+1}^{\kappa_{r}}\right] \gamma_{j}^{k+1}=0 \text { for } j=1, \ldots, r
$$

On the other hand the above recursion is again equivalent to the recursion (64) and (65) on the matrix polynomials $\Gamma_{k}(s)$. In fact, let us recall that, if $\boldsymbol{x}=\left[x_{0}, x_{1}, \ldots, x_{k}\right]^{T}$ represents the coefficients of the polynomial $x(s)$, then $\boldsymbol{x}^{\prime}:=\left[0, x_{0}, x_{1}, \ldots, x_{k}\right]^{T}$ represents the coefficients of $s x(s)$. Similarly, if the last entry of $\mathbf{x}$ vanishes, then also $\overrightarrow{\boldsymbol{x}}:=\left[0, x_{0}, x_{1}, \ldots, x_{k-1}\right]^{T}$ expresses the coefficients of $s x(s)$. Therefore, it is immediate to see that (64) is the polynomial versions of (66): now notice that, since $\Gamma_{k}(s)$ is an MF solution, $\operatorname{deg} \gamma_{i, j}^{k}(s)<\kappa_{i}$ if $i \neq j$, so the last entry of $\boldsymbol{\gamma}_{i, j}^{k}$ vanishes and thus (65) is the polynomial version of (67).

Now using the fact that $\kappa_{k+1}^{i}=\kappa_{k}^{i}$ for $i \neq j$ and $\kappa_{k+1}^{j}=\kappa_{k}^{j}+1$ and equation (68), Theorem 2 implies that $\Gamma_{k+1}(s)$ is a solution for the pair $\left(\mathcal{A}_{k+1}, W_{k+1}\right)$.

We have to check that the inequalities in (45) are also satisfied.

Consider first the elements in the $l^{\text {th }}$ column for $l \neq j$. If $\kappa_{k}^{l}<\kappa_{k}^{j}$ or $\kappa_{k}^{l}=\kappa_{k}^{j}$ and $l<j$, then $\epsilon_{k}^{l}=0$ and thus $\gamma_{i, l}^{k+1}(s)=\gamma_{i, l}^{k}(s)$ for $i=1, \ldots, r$, so the conditions in (45) are obviously satisfied. In particular, $\gamma_{l, l}^{k+1}(s)$ is monic and its degree is $\kappa_{k}^{l}=\kappa_{k+1}^{l}$.

If $\kappa_{k}^{l}>\kappa_{k}^{j}$ or $\kappa_{k}^{l}=\kappa_{k}^{j}$ but $j>l$ then

for $i \neq l$

$$
\begin{aligned}
\operatorname{deg} \gamma_{i, l}^{k+1}(s) & =\operatorname{deg}\left(\gamma_{i, l}^{k}(s)-\frac{\epsilon_{k+1}^{l}}{\epsilon_{k+1}^{j}} \gamma_{i, j}^{k}(s)\right) \leq \min \left(\kappa_{k}^{i}-1, \max \left(\kappa_{k}^{l}, \kappa_{k}^{j}\right)\right) \\
& =\min \left(\kappa_{k}^{i}-1, \kappa_{k}^{l}\right) \leq \min \left(\kappa_{k+1}^{i}-1, \kappa_{k+1}^{l}\right)
\end{aligned}
$$

for $i=l(45)$ yields

$$
\operatorname{deg} \gamma_{l, l}^{k+1}(s)=\operatorname{deg}\left(\gamma_{l, l}^{k}(s)-\frac{\epsilon_{k+1}^{l}}{\epsilon_{k+1}^{j}} \gamma_{l, j}^{k}(s)\right)=\kappa_{k}^{l}=\kappa_{k+1}^{l}
$$

and furthermore $\gamma_{l, l}^{k+1}(s)$ is monic; therefore, for these columns (45) holds.

Consider next the elements in the $j^{\text {th }}$ column. For $i=1, \ldots, r$,

$$
\gamma_{i, j}^{k+1}(s)=\left(s-\lambda_{k+1}\right) \gamma_{i, j}^{k}(s)-\sum_{l=0}^{r} \rho_{l, j}^{k} \gamma_{i, l}^{k+1}(s) .
$$

If $i=j$ then using the fact that $\operatorname{deg} \gamma_{i, j}^{k}(s) \leq \kappa_{k}^{i}-1$ for $i \neq j$ we obtain that

$$
\operatorname{deg} \gamma_{j, j}^{k+1}(s)=\operatorname{deg} s \gamma_{j, j}^{k+1}(s)=\kappa_{k}^{j}+1=\kappa_{k+1}^{j},
$$

moreover it is monic. 
Now if $i \neq j$ then we have to prove that

$$
\operatorname{deg} \gamma_{i, j}^{k+1}(s) \leq \min \left(\kappa_{k+1}^{i}-1, \kappa_{k+1}^{j}\right)
$$

If $i \neq j$ then $\operatorname{deg} s \gamma_{i, j}^{k}(s) \leq\left(\kappa_{k}^{i}-1\right)+1=\kappa_{k+1}^{i}$ but in the second term for $l \neq i$ $\operatorname{deg} \gamma_{i, l}^{k+1}(s) \leq \kappa_{k}^{i}-1$ while $\operatorname{deg} \gamma_{i, i}^{k+1}(s)=\kappa_{k}^{i}$, and thus this term has the highest degree. Moreover $\gamma_{i, i}^{k+1}(s)$ is monic; thus, if $\operatorname{deg} \gamma_{i, j}^{k}(s)=\kappa_{k}^{i}-1$, then the highest order term in $s \gamma_{i, j}^{k}(s)$ is canceled by the second sum. Consequently, for $i=1, \ldots, r, i \neq j$

$$
\operatorname{deg} \gamma_{i, j}^{k+1}(s)=\operatorname{deg}\left(s \gamma_{i, j}^{k}(s)-\sum_{l \neq j} \rho_{l, j}^{k} \gamma_{i, l}^{k+1}(s)\right) \leq \kappa_{k}^{i}-1=\kappa_{k+1}^{i}-1
$$

On the other hand, since, as we said, $\rho_{l, j}^{k}=0$ for $l: \kappa_{k}^{j}<\kappa_{k}^{l}-1$, we can write for $1 \leq i \leq r, i \neq j$

$$
\begin{aligned}
& \operatorname{deg} s \gamma_{i, j}^{k}(s) \leq \operatorname{deg} s \gamma_{j, j}^{k}(s)=\kappa_{k+1}^{j} \\
& \operatorname{deg} \rho_{l, j}^{k} \gamma_{i, l}^{k+1}(s) \leq \kappa_{k}^{l} \leq \kappa_{k}^{j}+1=\kappa_{k+1}^{j} \quad l \neq j
\end{aligned}
$$

which, together with (70), yields (69).

Let us point out again that the degrees of the columns of $\Gamma_{k+1}(s)$ are $\kappa_{k}^{l}=\kappa_{k+1}^{l}$ for $l \neq j$ and $\kappa_{k+1}^{j}=\kappa_{k}^{j}+1$. Thus it has degree $\sum_{l=1}^{r} \kappa_{k}^{l}+1=k+2$ and therefore it is minimal.

A multivariable recursive algorithm has been developed, for the special case of interpolating an $m \times p$ function $F(s)$ given its coefficients $F_{0}, \ldots, F_{k}$, in [13]. While it describes, in a behavioral framework, a clever generalization of [10], it also focuses only on a particular pair of interpolants; it presents therefore the same problem of jump in degree when this interpolating pair is not coprime.

\subsection{Tangential recursive interpolation algorithm}

While the proof of the algorithm is quite involved, its implementation is quite straightforward: we need, in fact only to compute (59), (66) and (67).

1. Initializing step: Let $i$ be the first column of $W$ whose first entry is not zero (such column exists, in view of controllability of $(\mathcal{A}, W)$ and lower triangularity of $\mathcal{A}$ ) and set

$$
\begin{array}{r}
W_{0}:=\left[0, \ldots, 0, w_{0}^{i}, \lambda_{0} w_{0}^{i}, w_{0}^{i+1}, \ldots, w_{0}^{r}\right] \\
\boldsymbol{\kappa}_{0}:=[0, \ldots, 0, \stackrel{i}{1}, 0, \ldots, 0]
\end{array}
$$


and

$$
\boldsymbol{\gamma}_{j}^{0}=\left[\begin{array}{c}
0 \\
\vdots \\
\hline-\frac{w_{0}^{j}}{w_{0}^{i}} \\
0 \\
\hline 0 \\
\vdots \\
1 \\
\vdots \\
0
\end{array}\right] j+1 \quad \text { if } j>i, \quad \boldsymbol{\gamma}_{j}^{0}=\left[\begin{array}{c}
0 \\
\vdots \\
1 \\
0 \\
\vdots \\
\frac{0}{0} \\
\frac{0}{0}
\end{array}\right] i \quad \text { if } j<i, \quad \boldsymbol{\gamma}_{i}^{0}=\left[\begin{array}{c}
0 \\
\vdots \\
\frac{0}{-\lambda_{00}} \\
\frac{1}{0} \\
\vdots \\
0
\end{array}\right] i
$$

2. Recursion step: Repeat while $k<K$ (where $K$ is the number of data):

- Given $\boldsymbol{W}_{k}^{\boldsymbol{\kappa}_{k}}, \boldsymbol{\gamma}_{j}^{k}$ for $j=1, \ldots, r$, compute $\boldsymbol{W}_{k+1}^{\boldsymbol{\kappa}_{k}}$ using (57) and $\boldsymbol{\epsilon}_{k+1}$ using (59). Set $i$ to be the index corresponding to the smallest $\kappa_{k}^{i}$ such that $\epsilon_{k+1}^{i}$ in (59) is different from 0 . Define, for $j=1, \ldots, r, \gamma_{j}^{k+1}$ using (66) and (67). Define $\boldsymbol{W}_{k+1}^{\boldsymbol{\kappa}_{k+1}}$ using (57) and (58).

- Increase $k$.

\section{Recursive interpolation of a given function-scalar case}

In the remaining part of this paper we consider a very special situation, when all the interpolation nodes are at the origin. We are going to show that this problem exhibit several interesting properties not present in the general case.

We suppose that the data $v_{0}, \ldots, v_{k}$ we consider are derived from the power series expansion of a given rational function

$$
f(s)=\frac{\beta(s)}{\alpha(s)}=\sum_{k=0}^{\infty} v_{k} s^{k},
$$

where we assume that $\alpha$ and $\beta$ are coprime, $\alpha$ is monic and that $\alpha(0) \neq 0$. We denote in this section by $p$ and $m$ the degrees of $\alpha$ and $\beta$, respectively.

We would like to assess now how the controllability indexes $\mu_{k}$ and $\nu_{k}$-giving the column degrees of minimal fundamental solutions-increase as the number of data $v_{0}, \ldots, v_{k}$ under consideration increases.

In this special scalar interpolation problem the matrices of the sequence of nested problems indexed by $k$, are given as 


$$
\mathcal{A}_{k}=\left[\begin{array}{cccc}
0 & \ldots & 0 & 0 \\
1 & 0 & \vdots & 0 \\
\ddots & 0 & \vdots \\
0 & \ldots & 1 & 0
\end{array}\right], \quad U_{k}=\left[\begin{array}{c}
1 \\
0 \\
\vdots \\
0
\end{array}\right], \quad V_{k}=\left[\begin{array}{c}
v_{0} \\
v_{1} \\
\vdots \\
v_{k}
\end{array}\right],
$$

where $\mathcal{A}_{k}$ is lower triangular $(k+1) \times(k+1)$ and $U_{k}$ and $V_{k}$ are $(k+1)$-dimensional vectors.

Due to the special structure of the matrix $\mathcal{A}$ the equations characterizing the polynomials $\beta^{U}, \alpha^{U}, \beta^{V}, \alpha^{V}$ can be written in a simpler form. (Since for the time being we analyze the properties for a fixed $k$, we omit the index from the notation.) Thus the matrix $U^{m}=\left[U, \mathcal{A} U, \mathcal{A}^{2} U, \ldots, \mathcal{A}^{m} U\right]$ is the matrix of dimension $(k+1) \times(m+1)$ of the form

$$
U^{m}=\left[\begin{array}{cccc}
1 & 0 & \ldots & 0 \\
0 & 1 & \ddots & 0 \\
\vdots & \ddots & \ddots & 0 \\
0 & \ldots & 0 & 1 \\
\vdots & & \ldots & 0 \\
\vdots & & \ddots & \vdots \\
0 & \ldots & & 0
\end{array}\right]
$$

Similarly, the matrix $V^{p}=\left[V, \mathcal{A} V, \mathcal{A}^{2} V, \ldots, \mathcal{A}^{p} V\right]$ is the matrix of dimension $(k+1) \times(p+1)$ of the form

$$
V^{p}=\left[\begin{array}{cccc}
v_{0} & 0 & \ldots & 0 \\
v_{1} & v_{0} & \ddots & 0 \\
\vdots & \ddots & \ddots & 0 \\
v_{p} & & v_{1} & v_{0} \\
v_{p+1} & v_{p-1} & \ldots & v_{1} \\
\vdots & & & \vdots \\
v_{k-1} & v_{k-2} & & v_{k-p-1} \\
v_{k} & v_{k-1} & \ldots & v_{k-p}
\end{array}\right]
$$

Notice that $U=U^{0}$ and $V=V^{0}$.

Denoting the controllability indexes of $U$ and $V$ by $\mu$ and $v$, respectively, we see that Eq. (16) characterizing the coefficient vectors of the polynomials in the minimal fundamental solution can be written as finding the kernel of the matrix $\left[U^{\mu},-V^{\nu}\right]$, which for $\mu \geq v$ can be written as follows: 


$$
\begin{aligned}
& {\left[U^{\mu},-V^{\nu}\right]}
\end{aligned}
$$

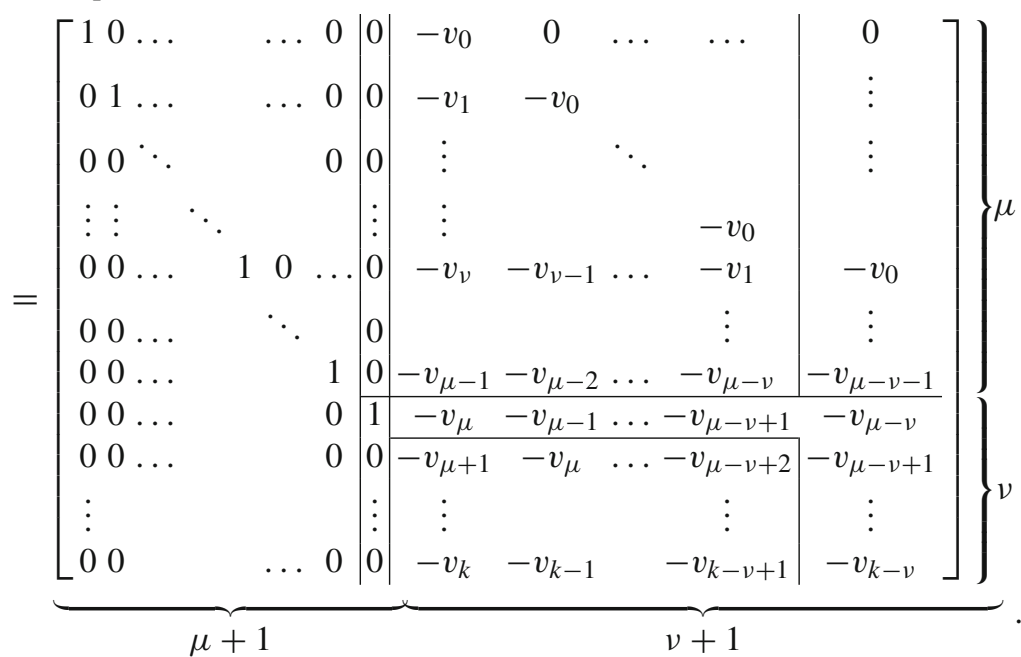

Let us point out that to determine $\mu$ the second block column should be written as a linear combination of the columns in the first and third block, while to find $v$ the last column should be expressed as a linear combination of all the previous ones. But the vectors in the first block are the unit vectors, thus for $\mu$ the part below the first horizontal line should be analyzed, for $v$ that one below the second horizontal line.

Using the special structure of (74) the behavior of the controllability indexes as $k \rightarrow \infty$ is described by the following Lemma.

Lemma 9 Given the rational function $f=\frac{\beta}{\alpha}$ define the $\mathcal{A}_{k}, U_{k}, V_{k}$ matrices as in (71). Denote by $\mu_{k}, v_{k}$ the controllability indexes of $\left(\mathcal{A}_{k},\left[U_{k}, V_{k}\right]\right)$. Let $\Gamma_{k}(s)=\left[\begin{array}{cc}\beta_{k}^{U} & \beta_{k}^{V} \\ \alpha_{k}^{U} & \alpha_{k}^{V}\end{array}\right]$ be the minimal fundamental solution of Problem 2 determined by $v_{0}, \ldots v_{k}$, for $k \geq 0$.

If $\operatorname{deg} \alpha \geq \operatorname{deg} \beta$, then $v_{k}=\operatorname{deg} \alpha$ for large enough $k$, and thus $\mu_{k}=k+1-v_{k} \rightarrow$ $\infty$ (when $k \rightarrow \infty)$, while if $\operatorname{deg} \alpha<\operatorname{deg} \beta$, then $\mu_{k}=\operatorname{deg} \beta$ when $k$ is large enough, and $v_{k} \rightarrow \infty($ as $k \rightarrow \infty)$.

Proof First let us assume that $\operatorname{deg} \alpha \geq \operatorname{deg} \beta$. That is, $f$ is a proper rational function.

In order to find the controllability index $v_{k}$ the last column in (74) should be expressed as a linear combination of the "previous" ones. But the columns in the first block part of (74) contains unit vectors this means that we should look after linear combination between the vectors in the third part-below the second horizontal line.

Now, let us denote by $p$ the degree of $\alpha$. Then computing from the power series expansion of $f=\frac{\beta}{\alpha}$ the coefficients of $s^{k}, k \geq p+1$, in $f(s) \alpha(s)$ we obtain that

$$
0=\alpha_{0} v_{p+l}+\alpha_{1} v_{p+l-1}+\cdots+\alpha_{p} v_{l} \text { for } l \geq 1
$$


Since $\alpha_{p} \neq 0$ the coefficient of the polynomial provides a linear combination we are looking for. Thus, we get if $k$ is large enough then $v_{k} \leq p$. Consequently, as $k \rightarrow \infty$ the sequence $v_{k}$ remains bounded, so $\mu_{k} \rightarrow \infty$.

At the same time, when we already have that $\mu_{k}>v_{k}$ then columns in the third block in the matrix (74) are linearly independent, thus the matrix

$$
\left[\begin{array}{cccc}
v_{v_{k}+1} & v_{v_{k}} & \ldots & v_{2} \\
v_{v_{k}+2} & v_{v_{k}+1} & \ldots & v_{3} \\
\vdots & \ddots & \ddots & \vdots \\
v_{k} & v_{k-1} & \ldots & v_{\mu_{k}}
\end{array}\right]
$$

is of full column-rank. Consequently, in this case $\alpha_{k}^{V}=\alpha$ and $\nu_{k}=\operatorname{deg} \alpha$.

Next consider the case when $p=\operatorname{deg} \alpha<\operatorname{deg} \beta$.

In order to find the controllability index $\mu_{k}$ the second column in (74) should be expressed as a linear combination of the "previous" ones. But the columns in the first block part of (74) contains unit vectors this means that we should look after linear combination expressing the vector in the second column using vectors from the third block column - but now below the first horizontal line.

Let us denote by $m$ the degree of $\beta$. Expressing again the coefficients of $s^{k}$ in $f(s) \alpha(s)$ but now for $k \geq m$ we obtain that

$$
\begin{aligned}
\beta_{m} & =\alpha_{0} v_{m}+\alpha_{1} v_{m-1}+\cdots+\alpha_{p} v_{m-p}, \\
0 & =\alpha_{0} v_{m+l}+\alpha_{1} v_{m+l-1}+\cdots+\alpha_{p} v_{m+l-p} \quad \text { for } l \geq 1
\end{aligned}
$$

Now since $\beta_{m} \neq 0$ we get that if $k$ is large enough then $\mu_{k} \leq m$. So $v_{k} \rightarrow \infty$ as $k \rightarrow \infty$.

If $v_{k}>\mu_{k}$, then the columns of the matrix

$$
\left[\begin{array}{cccc}
v_{\mu_{k}} & v_{\mu_{k}-1} & \ldots & v_{1} \\
v_{\mu_{k}+1} & v_{\mu_{k}} & \ldots & v_{2} \\
\vdots & \ddots & \ddots & \vdots \\
v_{k} & v_{k-1} & \ldots & v_{v_{k}}
\end{array}\right]
$$

are linearly independent and thus in this case $\alpha_{k}^{U}=\frac{1}{\beta_{p}} \alpha$ and $\mu_{k}=\operatorname{deg} \beta$. This concludes the proof of the Lemma.

In the construction of the recursive scheme for interpolation the so-called error terms $\epsilon^{U}, \epsilon^{V}$ played an important role.

Let $\left[U_{k+1}^{\mu_{k}},-V_{k+1}^{v_{k}}\right]$ be as in (25) and define the error terms arising from the linear combinations obtained in the $k^{\text {th }}$ step but including the next interpolation value $v_{k+1}$, as well 


$$
\begin{aligned}
{\left[\epsilon_{k+1}^{U}, \epsilon_{k+1}^{V}\right] } & :=\left[u_{k+1}^{\mu_{k}},-v_{k+1}^{v_{k}}\right]\left[\begin{array}{cc}
\boldsymbol{\beta}_{k}^{U} & \boldsymbol{\beta}_{k}^{V} \\
\boldsymbol{\alpha}_{k}^{U} & \boldsymbol{\alpha}_{k}^{V}
\end{array}\right] \\
& =\left[\sum_{j=0}^{\mu_{k}}\left(\beta_{k, j}^{U} u_{k-j+1}-\alpha_{k, j}^{U} v_{k-j+1}\right), \sum_{j=0}^{v_{k}}\left(\beta_{k, j}^{V} u_{k-j+1}-\alpha_{k, j}^{V} v_{k-j+1}\right)\right] .
\end{aligned}
$$

In general, given two polynomials $\beta_{k}(s), \alpha_{k}(s)$ of degree $\mu_{k}, v_{k}$, respectively, and the corresponding vectors $\boldsymbol{\beta}_{k}, \boldsymbol{\alpha}_{k}$, we can set $\epsilon_{k+1}:=u_{k+1}^{\mu_{k}} \boldsymbol{\beta}_{k}-v_{k+1}^{v_{k}} \boldsymbol{\alpha}_{k}$. The representation (44) in Corollary 1 implies that

$$
U_{k+1}^{\mu_{k}} \boldsymbol{\beta}_{k}-V_{k+1}^{v_{k}} \boldsymbol{\alpha}_{k}=\frac{1}{2 \pi i} \int_{\Delta}\left(s I-\mathcal{A}_{k+1}\right)^{-1}\left[U_{k+1} \beta_{k}(s)-V_{k+1} \alpha_{k}(s)\right] d s .
$$

The last row of the vector on the left hand side is $\epsilon_{k+1}$; thus, using that the last row of $(s I-\mathcal{A})^{-1}$ in this special interpolation problem is $\left[s^{-(k+1)}, s^{-k}, \ldots, s^{-1}\right]$ we can write that

$$
\epsilon_{k+1}=\frac{1}{2 \pi i} \int_{\Delta} \sum_{i=0}^{k+1} s^{-i-1}\left[u_{k+1-i} \beta_{k}(s)-v_{k+1-i} \alpha_{k}(s)\right] d s .
$$

Corollary 2 Suppose $\beta_{k}(s)=s \beta_{k-1}(s)$ and $\alpha_{k}(s)=s \alpha_{k-1}(s)$. Then

$$
\epsilon_{k+1}=\epsilon_{k}
$$

Proof From (78), we have:

$$
\begin{aligned}
\epsilon_{k+1}= & \frac{1}{2 \pi i} \int_{\Delta} \sum_{i=0}^{k+1} s^{-i-1}\left[u_{k+1-i} \beta_{k}(s)-v_{k+1-i} \alpha_{k}(s)\right] d s \\
= & \frac{1}{2 \pi i} \int_{\Delta} \sum_{i=0}^{k+1} s^{-i-1}\left[u_{k+1-i} s \beta_{k-1}(s)-v_{k+1-i} s \alpha_{k-1}(s)\right] d s \\
= & \frac{1}{2 \pi i} \int_{\Delta} \sum_{i=1}^{k+1} s^{-i}\left[u_{k+1-i} \beta_{k-1}(s)-v_{k+1-i} \alpha_{k-1}(s)\right] d s \\
& +\frac{1}{2 \pi i} \int_{\Delta}\left[u_{k+1} \beta_{k-1}(s)-v_{k+1} \alpha_{k-1}(s)\right] d s \\
= & \frac{1}{2 \pi i} \int_{\Delta} \sum_{i=0}^{k} s^{-i-1}\left[u_{k-i} \beta_{k-1}(s)-v_{k-i} \alpha_{k-1}(s)\right] d s=\epsilon_{k} .
\end{aligned}
$$


In the general recursion scheme presented in Theorem 1 the minimal fundamental solution $\Gamma_{k+1}$ was defined using $\Gamma_{k}$. In the present situation-as we are going to seeit will be natural to consider larger steps. To this end, given $k_{1}<k_{2}$ and the associated minimal fundamental solutions $\Gamma_{k_{1}}(s)$ and $\Gamma_{k_{2}}(s)$, set $\tilde{\Gamma}_{k_{1}, k_{2}}(s):=\Gamma_{k_{1}}^{-1}(s) \Gamma_{k_{2}}(s)$ (it clearly is a polynomial matrix). If $k_{2}=k_{1}+1$ then we write shortly $\tilde{\Gamma}_{k_{2}}(s)=\tilde{\Gamma}_{k_{1}, k_{2}}(s)$.

In the generic case, for each $k$, the errors $\epsilon_{k}^{U}, \epsilon_{k}^{V}$ are both nonzero and, since $\mu_{k}+v_{k}=k+1$, from Theorem 1 we obtain that $\mu_{k}=v_{k}$ for $k$ odd and $\mu_{k}=v_{k}+1$ for $k$ even. Graphically, the representation is the following:

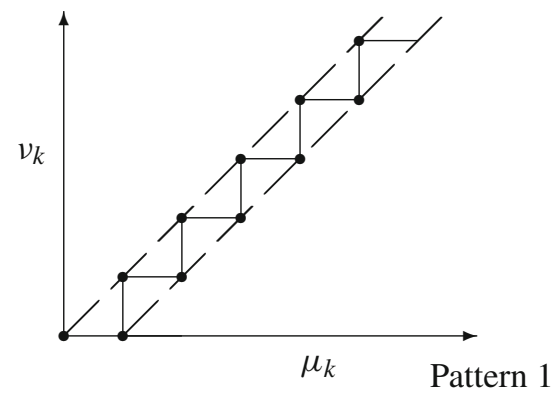

So, if $\mu_{k}=v_{k}$ and $\epsilon_{k+1}^{U} \neq 0$, following Theorem 1, we will use (38) to interpolate also the $(k+1)$-st data $v_{k+1}$. If also $\epsilon_{k+2}^{V} \neq 0$, then we can then use (39) to match $v_{k+2}$. Notice that, if $\epsilon_{k+1}^{V}=0$, then $\tilde{\Gamma}_{k, k+1}(s)$ (the last factor in (38)) is lower triangular. It is not hard to see that, in this case, $\operatorname{deg} \beta_{k+1}^{U}(s)>\operatorname{deg} \beta_{k+1}^{V}+1$ and thus $\beta_{k+1, \mu_{k+1}-1}^{V}=0$ : that is, also $\tilde{\Gamma}_{k+1, k+2}(s)$ is lower triangular. In conclusion, if $\mu_{k}=v_{k}$ and $\epsilon_{k+1}^{V}=0$, then $\Gamma_{k+2}(s)$ and $\Gamma_{k}(s)$ are related by a lower triangular matrix. Similarly, if $\mu_{k}=$ $v_{k}+1$ and both $\epsilon_{k+1}^{V}$ and $\epsilon_{k+2}^{U}$ are not zero, we can use (39) and (38) to match the next two data $v_{k+1}, v_{k+2}$. If $\epsilon_{k+1}^{U}=0$, then $\Gamma_{k+2}(s)$ and $\Gamma_{k}(s)$ are related, this time, by an upper triangular matrix.

What is surprising is that this is true whenever one of the errors is 0 , even as we go astray from the pattern 1 , as the next result shows.

Theorem 4 Consider the recursive version of Problem 2 determined by the coefficients in the Taylor-series expansion of the rational function $f(s)$ considered around the origin.

(i) Suppose that for some $k$ we have that $\mu_{k}=v_{k}+1$ and $\epsilon_{k+1}^{V}=\cdots=\epsilon_{k+l}^{V}=0$ and $\epsilon_{k+l+1}^{V} \neq 0$.

Let $k^{\prime}:=k+2 l$ : then $\mu_{k^{\prime}}=v_{k^{\prime}}+1$ and $k^{\prime}$ is the next smallest integer with this property. Furthermore, the matrix $\tilde{\Gamma}_{k, k^{\prime}}$ relating $\Gamma_{k}$ and $\Gamma_{k^{\prime}}$ from repeated iterations of (38) and (39) is lower triangular. That is,

$$
\Gamma_{k^{\prime}}(s)=\left[\begin{array}{cc}
\beta_{k^{\prime}}^{U} & \beta_{k^{\prime}}^{V} \\
\alpha_{k^{\prime}}^{U}, & \alpha_{k^{\prime}}^{V}
\end{array}\right]=\left[\begin{array}{cc}
\beta_{k}^{U} & \beta_{k}^{V} \\
\alpha_{k}^{U} & \alpha_{k}^{V}
\end{array}\right]\left[\begin{array}{cc}
s^{l} & 0 \\
p_{l}(s) & s^{l}
\end{array}\right]=\Gamma_{k}(s) \tilde{\Gamma}_{k, k^{\prime}}(s),
$$


where $l=v_{k^{\prime}}-v_{k}$ and $p_{l}$ is suitable polynomial of degree less than $l$.

(ii) Similarly, suppose $\mu_{k}=v_{k}$ and $\epsilon_{k+1}^{U}=\cdots=\epsilon_{k+l}^{U}=0$ and $\epsilon_{k+l+1}^{U} \neq 0$.

Let $k^{\prime}:=k+2 l$ : then $\mu_{k^{\prime}}=v_{k^{\prime}}$ and $k^{\prime}$ is the next smallest integer with this property. Moreover, the matrix $\tilde{\Gamma}_{k, k^{\prime}}$ relating $\Gamma_{k}$ and $\Gamma_{k^{\prime}}$ from repeated iterations of (38) and (39) is upper triangular. That is,

$$
\Gamma_{k^{\prime}}(s)=\left[\begin{array}{cc}
\beta_{k^{\prime}}^{U} & \beta_{k^{\prime}}^{V} \\
\alpha_{k^{\prime}}^{U}, & \alpha_{k^{\prime}}^{V}
\end{array}\right]=\left[\begin{array}{cc}
\beta_{k}^{U} & \beta_{k}^{V} \\
\alpha_{k}^{U} & \alpha_{k}^{V}
\end{array}\right]\left[\begin{array}{cc}
s^{l} & p_{l}(s) \\
0 & s^{l}
\end{array}\right]=\Gamma_{k}(s) \tilde{\Gamma}_{k, k^{\prime}}(s),
$$

where $l=\mu_{k^{\prime}}-\mu_{k}$, where $p_{l}$ is a suitable polynomial of degree no greater than $l$.

Proof Since $\mu_{k}=v_{k}+1>v_{k}$, but $\epsilon_{k+1}^{V}=\cdots=\epsilon_{k+l}^{V}=0$, in accordance with Theorem 1, we need, in order to obtain $\Gamma_{k, k^{\prime}}$, to use first (38) $l$ times. It is immediate to verify that each of these factors is lower triangular. Thus also the matrix relating $\Gamma_{k}$ and $\Gamma_{k+l}$ is lower triangular. Furthermore, during these steps the second controllability index does not change, that is, $v_{k+l}=v_{k}$, while the first one in each step increases by one: $\mu_{k+l}=\mu_{k}+l$.

We claim now that, to obtain $\Gamma_{k^{\prime}}$, we need $l$ iterations of (39) which are also lower triangular. Since $\epsilon_{k+l+1}^{V} \neq 0$, for the step $k+l+1$ we have to use (39). Now the column degrees of $\Gamma_{k+l}$ are $v_{k}+l+1, v_{k}$. Therefore, $\operatorname{deg} \beta_{k+l}^{U}-\operatorname{deg} \beta_{k+l}^{V} \geq l+1$ and thus the coefficient $\beta_{k+l, \mu_{k+l}-1}^{V}=0$; consequently, also the last factor in (39) is lower triangular for this step. Thus, $\beta_{k+l+1}^{V}(s)=s \beta^{V}(s)_{k+l}$ and $\alpha_{k+l+1}^{V}(s)=s \alpha_{k+l}^{V}(s)$ In view of Corollary $2, \epsilon_{k+l+2}^{V}=\epsilon_{k+l+1}^{V}$. Thus it is different from zero. The same reasoning can be repeated while $\tilde{\Gamma}_{k+l+j+1}(s)$ is lower triangular, that is as long as $\operatorname{deg} \beta_{k+l+j}^{U}-\operatorname{deg} \beta_{k+l+j}^{V}>1$. This will happen, if $\mu_{k+l+j}>v_{k+l+j}+1$. But during these steps the sequence of $\mu$-s does not change. Thus

$$
\mu_{k+l+j}=v_{k}+l+1, \quad v_{k+l+j}=v_{k}+j .
$$

$\tilde{\Gamma}_{k+l+j+1}(s)$ still will be lower triangular for $j=0, \ldots, l-1$. In conclusion, $\tilde{\Gamma}_{k, k^{\prime}}(s)$ is lower triangular. Since it has determinant $2 l$, and each column has degree at least $l$, it can only have the form described in (79).

Let us point out that using again Corollary 2 for $\epsilon_{k^{\prime}}^{V}$ we obtain that $\epsilon_{k^{\prime}+1}^{V} \neq 0$ and thus $\mu_{k^{\prime}+1}=\mu_{k^{\prime}}$ and $v_{k^{\prime}+1}=v_{k^{\prime}}+1$. But now the factor $\tilde{\Gamma}_{k^{\prime}+1}$ is not necessarily lower triangular.

The second statement can be proven similarly. But in this case the final application of Corollary 2 gives that $\mu_{k^{\prime}+1}=\mu_{k^{\prime}}+1$ and $v_{k^{\prime}+1}=v_{k^{\prime}}$ again pointing out that now the polynomial matrix $\tilde{\Gamma}_{k^{\prime}+1}(s)$ is not necessarily upper triangular.

In conclusion, the possible paths the controllability indexes can exhibit are of the forms: 

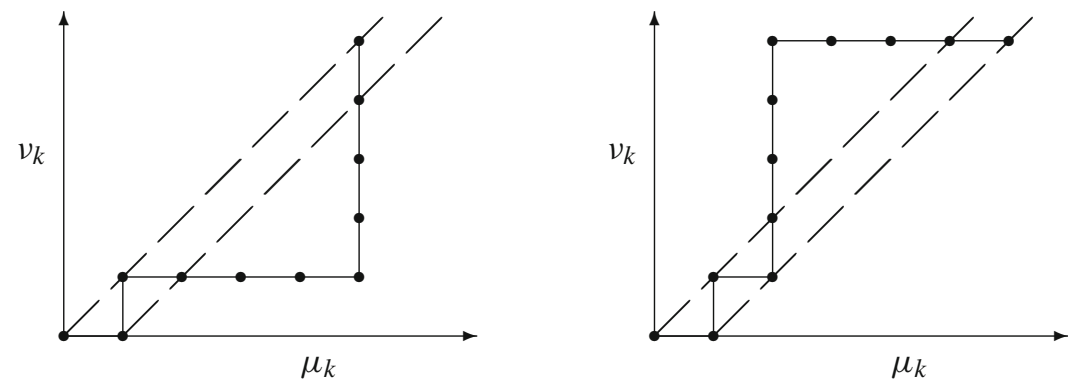

Paths of the following form are not admissible:
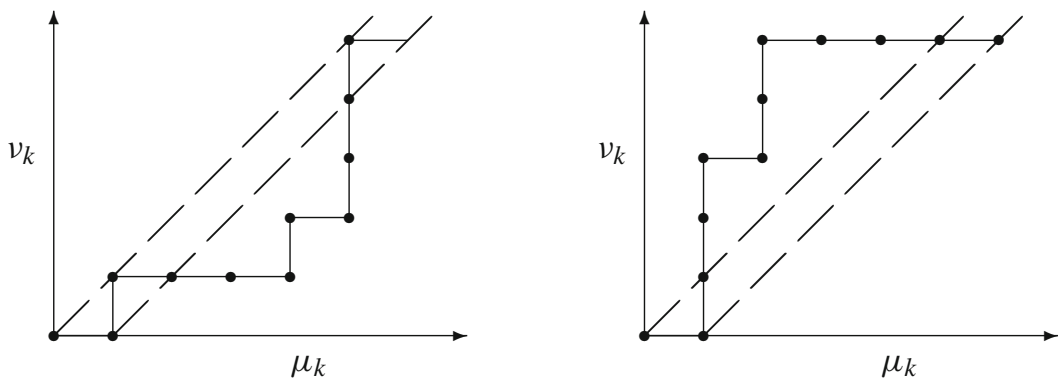

The construction and the proof of Theorem 4 is based on Theorem 1 determining a recursion for the minimal fundamental solutions of Problem 2. The next theorem is based on a recursion for an appropriate sequence of fundamental (not necessarily minimal) solutions. This construction gives the possibility of explicitly determining the positions in the jumps of the degrees in the minimal solutions of the interpolation problem using the form of the given rational function $f$. The basic idea is that if for example $\beta^{V}, \alpha^{V}$ are already given and provide a solution for Problem 2 for $v_{0}, v_{1}, \ldots, v_{K}$, then to find the corresponding $\beta^{U}, \alpha^{U}$ which complete the pair to a minimal solution, a pair of polynomials should be determined in such a way that the determinant of the corresponding polynomial matrix formed from these polynomial vectors is equal to $s^{K+1}$.

In order to show this let us assume that $f=\frac{\beta}{\alpha}$, where $\operatorname{deg} \alpha=p, \operatorname{deg} \beta=m$ and $\alpha(0) \neq 0$, and generate a sequence of polynomials using the following recursion:

First let us denote by $h_{1} \geq 0$ the multiplicity of 0 as a possible zero of $\beta$ and introduce the notation: $\beta_{1}(s) s^{\bar{h}_{1}}=\beta(s)$. (That is $\beta_{1}(0) \neq 0$.) Consider the solutions of the following equation

$$
\alpha(s)=\gamma_{1}(s) \beta_{1}(s)-s^{h_{1}} \delta_{1}(s)
$$

where $\gamma_{1}$ and $\delta_{1}$ are polynomials, $\operatorname{deg} \gamma_{1} \leq h_{1}$, while $\delta_{1}(0)=0$. Notice that the coefficients of $1, s, \ldots, s^{h_{1}}$ in $s^{h_{1}} \delta_{1}$ are zero. Since according to the construction the constant term in $\beta_{1}$ is nonzero, this equation has a unique solution. Furthermore,

$$
\operatorname{deg} \delta_{1} \leq \max \left(\operatorname{deg} \alpha-h_{1}, \operatorname{deg} \beta_{1}\right)
$$


Let us continue similarly — as in the Euclidean-algorithm-denoting by $h_{2} \geq 1$ the multiplicity of 0 as the zero of $\delta_{1}$ and introducing the polynomial $\beta_{2}$ as

$$
\beta_{2}(s) s^{h_{2}}=\delta_{1}(s)
$$

solve the equation

$$
\beta_{1}(s)=\gamma_{2}(s) \beta_{2}(s)-s^{h_{2}} \delta_{2}(s)
$$

where $\gamma_{2}, \delta_{2}$ are polynomials, $\operatorname{deg} \gamma_{2} \leq h_{2}$ and $\delta_{2}(0)=0$. In general, if

$$
\beta_{j-1}(s)=\gamma_{j}(s) \beta_{j}(s)-s^{h_{j}} \delta_{j}(s),
$$

where $\delta_{j}(0)=0$, then denote by $h_{j+1}$ the multiplicity of 0 as the zero of $\delta_{j}$ and set

$$
\beta_{j+1}(s) s^{h_{j+1}}=\delta_{j}(s) .
$$

Then $\gamma_{j+1}$ and $\delta_{j+1}$ are defined as solutions of

$$
\beta_{j}(s)=\gamma_{j+1}(s) \beta_{j+1}(s)-s^{h_{j+1}} \delta_{j+1}(s),
$$

where $\operatorname{deg} \gamma_{j+1} \leq h_{j+1}$ and $\delta_{j+1}(0)=0$. We obtain that

$$
\operatorname{deg} \beta_{j+1}=\operatorname{deg} \delta_{j+1}-h_{j+1}<\operatorname{deg} \delta_{j+1} \leq \max \left(\operatorname{deg} \beta_{j-1}-h_{j}, \operatorname{deg} \beta_{j}\right) .
$$

Consequently, the sequence $\operatorname{deg} \beta_{j}, j \geq 2$ is strictly decreasing, so this algorithm terminates in finite steps. That is, for some $r$ we have that

$$
\beta_{r-1}(s)=\gamma_{r}(s) \beta_{r}(s)
$$

Straightforward computation gives that

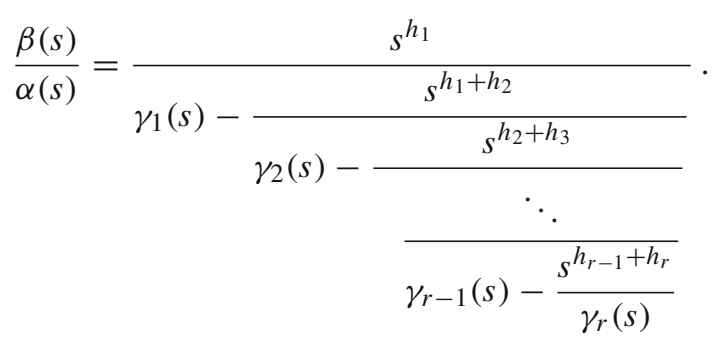

Now let us define a sequence a polynomial pairs as follows:

$$
\begin{aligned}
& {\left[\begin{array}{l}
N_{0}(s) \\
R_{0}(s)
\end{array}\right]=\left[\begin{array}{l}
0 \\
1
\end{array}\right] ;} \\
& {\left[\begin{array}{l}
N_{j}(s) \\
R_{j}(s)
\end{array}\right]=\left[\begin{array}{cc}
0 & s^{h_{1}} \\
-s^{h_{1}} & \gamma_{1}(s)
\end{array}\right] \cdots\left[\begin{array}{cc}
0 & s^{h_{j}} \\
-s^{h_{j}} & \gamma_{j}(s)
\end{array}\right]\left[\begin{array}{l}
0 \\
1
\end{array}\right] .}
\end{aligned}
$$


for $j=1, \ldots, r$. We obtain that

$$
\left[\begin{array}{ll}
-s^{h_{j}} N_{j-1}(s) & N_{j}(s) \\
-s^{h_{j}} R_{j-1}(s) & R_{j}(s)
\end{array}\right]=\left[\begin{array}{cc}
0 & s^{h_{1}} \\
-s^{h_{1}} & \gamma_{1}(s)
\end{array}\right] \cdots\left[\begin{array}{cc}
0 & s^{h_{j}} \\
-s^{h_{j}} & \gamma_{j}(s)
\end{array}\right] .
$$

and

$$
\frac{N_{j}(s)}{R_{j}(s)}=\frac{s^{h_{1}}}{\gamma_{1}(s)-\frac{s^{h_{1}+h_{2}}}{\gamma_{2}(s)-\frac{s^{h_{3}}}{\gamma_{j-1}(s)-\frac{s^{h_{j-1}+h_{j}}}{\gamma_{j}(s)}}}} .
$$

In particular,

$$
\frac{N_{r}(s)}{R_{r}(s)}=\frac{\beta(s)}{\alpha(s)}
$$

Let us introduce the notation

$$
l_{j}=\sum_{i=1}^{j} h_{i}
$$

For the sake of simplicity we are going to assume that $h_{1} \geq 1$. In this case $h_{1}=$ $\min \left\{h \mid v_{h} \neq 0\right\}$.

Let us point out that if $h_{1}=0$ then $h_{2}=\min \left\{h \geq 1 \mid v_{h} \neq 0\right\}$, thus the sequence of $l_{1}, l_{2}, \ldots$ essentially remains the same, except the 0 as a first element is added to it.

Theorem 5 Assume that $f(s)=\frac{\beta(s)}{\alpha(s)}$, where $\alpha(0) \neq 0$ while $\beta(0)=0$, with the Taylor-series expansion around the origin

$$
f(s)=\sum_{j=1}^{\infty} v_{j} s^{j}
$$

Apply the construction described above to obtain the polynomials $N_{j}, R_{j}$ for $j=$ $1, \ldots, r$. Then a fundamental solution for $K=2 l_{j}-1$ is given by

$$
\left[\begin{array}{ll}
-s^{h_{j}} N_{j-1} & N_{j} \\
-s^{h_{j}} R_{j-1} & R_{j}
\end{array}\right]
$$

Furthermore, both controllability indexes $\mu_{k}, v_{K}$ are equal to $l_{j}$, and coincide with the column degrees of this matrix.

Proof Let us note that the assumption $\beta(0)=0$ implies that $h_{1} \geq 1$ and representation (82) implies that $\operatorname{deg} R_{j} \leq l_{j}, \operatorname{deg} N_{j} \leq l_{j}$ 
First we are going to show by induction according to the number of products in the representation (82) that the polynomials $N_{j}, R_{j}$ are coprime, and $R_{j}(0) \neq 0$. Let us observe that since according to the construction $\beta_{j-1}(0) \neq 0, \beta_{j}(0) \neq 0$ while $\delta_{j}(0)=0$ we have from Eq. (81) that $\gamma_{j}(0) \neq 0$. Thus $s^{h_{j}}$ and $\gamma_{j}$ are coprime. Now let us introduce the notation

$$
\left[\begin{array}{c}
N_{j}^{(2)}(s) \\
R_{j}^{(2)}(s)
\end{array}\right]=\left[\begin{array}{cc}
0 & s^{h_{2}} \\
-s^{h_{2}} & \gamma_{2}(s)
\end{array}\right] \cdots\left[\begin{array}{cc}
0 & s^{h_{j}} \\
-s^{h_{j}} & \gamma_{j}(s)
\end{array}\right]\left[\begin{array}{l}
0 \\
1
\end{array}\right]
$$

for $j=2, \ldots, r$. Let us observe that $N_{j}^{(2)}(0)=0$ while $R_{j}^{(2)}(0) \neq 0$.

Then $N_{j}$ and $R_{j}$ can be expressed as

$$
\begin{aligned}
& N_{j}(s)=s^{h_{1}} R_{j}^{(2)}(s), \\
& R_{j}(s)=-s^{h_{1}} N_{j}^{(2)}(s)+\gamma_{j}(s) R_{j}^{(2)}(s),
\end{aligned}
$$

where $N_{j}^{(2)}$ and $R_{j}^{(2)}$ are coprime using the induction hypothesis, while $\gamma_{j}(0) \neq 0$ implying that $N_{j}$ and $R_{j}$ are coprime, as well. At the same time, since $N_{j}^{(2)}(0)=0$ we obtain that $R_{j}(0) \neq 0$ using the corresponding induction hypothesis on $R_{j}^{(2)}$.

The representation (83) gives that

$$
\operatorname{det}\left[\begin{array}{ll}
-s^{h_{j+1}} N_{j}(s) & N_{j+1}(s) \\
-s^{h_{j+1}} R_{j}(s) & R_{j+1}(s)
\end{array}\right]=s^{2 l_{j+1}} .
$$

Consequently,

$$
\frac{N_{j}(s)}{R_{j}(s)}-\frac{N_{j+1}(s)}{R_{j+1}(s)}=\frac{s^{2 l_{j}+h_{j+1}}}{R_{j}(s) R_{j+1}(s)},
$$

implying the $\frac{N_{j}}{R_{j}}$ is a solution of the Interpolation Problem 2 determined by the values $v_{0}=0, v_{1}, \ldots, v_{l_{j}+l_{j+1}-1}$. In particular, the multiplicity of the origin as a zero of $N_{j}(s)$ is exactly $h_{1}$.

Notice that the pair $s^{h_{j}} N_{j-1}(s), s^{h_{j}} R_{j-1}(s)$ provides a solution for the Problem 2 determined by the values $v_{0}=0, v_{1}, \ldots, v_{2 l_{j}-1}$.

Choosing $K=2 l_{j}-1$ we obtain that both columns of the matrix

$$
\left[\begin{array}{ll}
-s^{h_{j}} N_{j-1}(s) & N_{j}(s) \\
-s^{h_{j}} R_{j-1}(s) & R_{j}(s)
\end{array}\right]
$$

provide solutions of the Problem 2 for $v_{0}, v_{1}, \ldots, v_{K}$ and the determinant of this matrix equals to that of the minimal fundamental solution $\Gamma_{K}(s)$. Thus

$$
\Gamma_{K}=\left[\begin{array}{ll}
-s^{h_{j}} N_{j-1}(s) & N_{j}(s) \\
-s^{h_{j}} R_{j-1}(s) & R_{j}(s)
\end{array}\right]\left(\Gamma_{K}^{-1}\left[\begin{array}{ll}
-s^{h_{j}} N_{j-1}(s) & N_{j}(s) \\
-s^{h_{j}} R_{j-1}(s) & R_{j}(s)
\end{array}\right]\right)^{-1}
$$


where the matrix standing in the brackets is unimodular, thus its inverse is a polynomial matrix. Consequently, the matrix (84) is a fundamental solution, as well, concluding the proof of the first part.

Furthermore, since the column degrees of the matrix (83) are no greater than $l_{j}$ while its determinant is $s^{2 l_{j}}$, its highest column degree coefficient matrix should be of full rank and both column degrees should equal to $l_{j}$. Using Lemma 6.3-14 in Kailath [12] we obtain that its column degrees and those of $\Gamma_{K}$ coincide. Thus $l_{j}=v_{K}$ and $\mu_{K}=K+1-v_{K}=v_{K}$.

Remark 8 In order to get the minimal fundamental solution $\Gamma_{K}$ from the matrix (84) the degrees of the $(1,2)$ and $(2,1)$ elements should be reduced and the $(1,1)$ and $(2,2)$ elements should be transformed to monic polynomials. Denoting by $g_{j}$ and $f_{j}$ the coefficients of $s^{l_{j}}$ in $R_{j}(s)$ and $N_{j}(s)$, respectively, Eq. (86) implies that

$$
\operatorname{det}\left[\begin{array}{ll}
-f_{j-1} & f_{j} \\
-g_{j-1} & g_{j}
\end{array}\right]=1 .
$$

Consequently,

$$
\begin{aligned}
\Gamma_{K}(s)= & {\left[\begin{array}{cc}
\beta_{K}^{U}(s) & \beta_{K}^{V}(s) \\
\alpha_{K}^{U}(s) & \alpha_{K}^{V}(s)
\end{array}\right] } \\
& =\left[\begin{array}{ll}
-s^{h_{j}} N_{j-1}(s) & N_{j}(s) \\
-s^{h_{j}} R_{j-1}(s) & R_{j}(s)
\end{array}\right]\left[\begin{array}{ll}
-f_{j-1} & f_{j} \\
-g_{j-1} & g_{j}
\end{array}\right]^{-1} \\
& =\left[\begin{array}{ll}
-s^{h_{j}} N_{j-1}(s) & N_{j}(s) \\
-s^{h_{j}} R_{j-1}(s) & R_{j}(s)
\end{array}\right]\left[\begin{array}{cc}
g_{j} & -f_{j} \\
g_{j-1} & -f_{j-1}
\end{array}\right],
\end{aligned}
$$

where $K=2 l_{j}-1$.

Now, combining Theorems 4 and 5 we can describe the paths of the controllability indexes generated by the minimal fundamental solutions to the restricted interpolation problems generated by the Taylor-series of a given function $f$. In fact, using the notations of Theorem 5 the following theorem holds.

Theorem 6 Let introduce the notation $k_{j}=2 l_{j}-1$. Then the set

$$
\left\{k_{j} \mid j \geq 1\right\}
$$

coincides with the set containing those values when for the recursive version of Problem 2 determined by the Taylor-series coefficients of the function $f=\frac{\beta}{\alpha}$ the controllability indexes coincide. In particular, $\mu_{k_{j}}=v_{k_{j}}=l_{j}$.

Furthermore,

(i) if the coefficient $g_{j}$ of the term $s^{l_{j}}$ in $R_{j}$ is zero, then

$$
\mu_{k_{j}+1}=\mu_{k_{j}}, \quad v_{k_{j}+1}=v_{k_{j}}+1
$$

and the path of the pair of controllability indexes evolves according to part (i) of Theorem 4. 
(ii) if $g_{j} \neq 0$, then

$$
\mu_{k_{j}+1}=\mu_{k_{j}}+1, \quad v_{k_{j}+1}=v_{k_{j}} .
$$

\section{If moreover}

(a) $h_{j+1}=1$ then in the next step both controllability indexes are equal:

$$
\mu_{k_{j}+2}=v_{k_{j}+2}=l_{j+1} \text {. }
$$

(This is the generic case.)

(b) If $g_{j} \neq 0$ and $h_{j+1}>1$ then the path determined by the controllability indexes evolves according to part (ii) of Theorem 4.

Proof Theorem 5 implies that $\mu_{k_{j}}=v_{k_{j}}=l_{j}$. Analyzing the path behavior of the controllability indexes we show that the next occasion when they are equal again is given by $l_{j+1}$, moreover at the same time we describe the form of the path between these two points.

From Theorem 5 we have obtained that

$$
\begin{gathered}
\beta_{k_{j}}^{U}(s)=-g_{j} s^{h_{j}} N_{j-1}(s)+g_{j-1} N_{j}(s) \\
\alpha_{k_{j}}^{U}(s)=-g_{j} s^{h_{j}} R_{j-1}(s)+g_{j-1} R_{j}(s) .
\end{gathered}
$$

It was already pointed out that the pair $N_{j}, R_{j}$ provide solutions to the Problem 2 exactly up to $l_{j}+l_{j+1}-1$. Thus, if the next interpolation value $v_{l_{j}+l_{j+1}}$ is taken into consideration there will be a nonzero error term obtained via using this pair of polynomials. Let us denote this error by $\epsilon_{j}$. That is,

$$
\epsilon_{j}:=u_{l_{j}+l_{j+1}}^{\mu_{l_{j}+l_{j+1}-1}} \boldsymbol{N}_{j}-v_{l_{j}+l_{j+1}}^{\nu_{l_{j}+l_{j+1}-1}} \boldsymbol{R}_{j} .
$$

Since $h_{j+1} \geq 1$ and thus $l_{j}+l_{j+1}-1 \geq 2 l_{j}$ so the pair $N_{j}, R_{j}$ still interpolates for the next value $v_{2 l_{j}}$, using Corollary 2 we obtain that

$$
\epsilon_{k_{j}+1}^{U}=-g_{j-1} \epsilon_{j-1}
$$

Similar computation gives that

$$
\epsilon_{k_{j}+1}^{V}=f_{j} \epsilon_{j-1}
$$

Consequently, $\epsilon_{k_{j}+1}^{U}=0$ if and only if $g_{j}=0$. Theorem 1 implies that in this case $\mu_{k_{j}+1}=\mu_{k_{j}}$, and $v_{k_{j}+1}=v_{k_{j}}+1$. But if $g_{j}=0$ then the pair $\beta_{k_{j}}^{U}, \alpha_{k_{j}}^{U}$ are obtained from $N_{j}, R_{j}$ via multiplication with a nonzero constant $g_{j-1}$, thus part ii) of Theorem 1 gives that in the present case $\beta_{k_{j}+1}^{U}=\beta_{k_{j}}^{U}, \alpha_{k_{j}+1}^{U}=\alpha_{k_{j}}^{U}$ providing solutions to the interpolation problem determined by $v_{0}, v_{1}, \ldots, v_{k}$ up to $k \leq 2 l_{j}+h_{j+1}-1$. Now 
the second part of Theorem 4 gives that for the next $h_{j+1}$ steps $v$ remains constant while $\mu$ increases. That is

$$
\begin{aligned}
v_{k_{j}+h_{j+1}+l} & =v_{k_{j}+h_{j+1}}=l_{j}+h_{j+1}, \quad \text { for } l=0, \ldots, h_{j+1}, \\
\mu_{k_{j}+h_{j+1}+l} & =\mu_{k_{j}}+l=l_{j}+l, \quad \text { for } l=0, \ldots, h_{j+1} .
\end{aligned}
$$

In particular, $\mu_{k_{j+1}}=v_{k_{j+1}}=l_{j+1}$ proving that in this case $l_{j+1}$ is the next value when the controllability indexes are equal, and also describing the path behavior of this pair of indexes, concluding the proof of part i).

Now, if $g_{j} \neq 0$, then from Theorem 1 we obtain that $\mu_{k_{j}+1}=\mu_{k_{j}}+1=l_{j}+1$, $v_{k_{j}+1}=v_{k_{j}}=l_{j}$ and

$$
\begin{aligned}
& \beta_{k_{j}+1}^{V}=-\frac{\epsilon_{k_{j}+1}^{V}}{\epsilon_{k_{j}+1}^{U}} \beta_{k_{j}}^{U}+\beta_{k_{j}}^{V}, \\
& \alpha_{k_{j}+1}^{V}=-\frac{\epsilon_{k_{j}+1}^{V}}{\epsilon_{k_{j}+1}^{U}} \alpha_{k_{j}}^{U}+\alpha_{k_{j}}^{V} .
\end{aligned}
$$

Straightforward calculation based on part i) of Theorem 1 gives that

$$
\begin{aligned}
& \beta_{k_{j}+2}^{V}=s^{h_{j}} R_{j-1}\left(f_{j}+g_{j} \frac{\epsilon_{k_{j}+1}^{V}}{\epsilon_{k_{j}+1}^{U}}\right)-R_{j}\left(f_{j-1}+g_{j-1} \frac{\epsilon_{k_{j}+1}^{V}}{\epsilon_{k_{j}+1}^{U}}\right), \\
& \alpha_{k_{j}+2}^{V}=s^{h_{j}} N_{j-1}\left(f_{j}+g_{j} \frac{\epsilon_{k_{j}+1}^{V}}{\epsilon_{k_{j}+1}^{U}}\right)-N_{j}\left(f_{j-1}+g_{j-1} \frac{\epsilon_{k_{j}+1}^{V}}{\epsilon_{k_{j}+1}^{U}}\right) .
\end{aligned}
$$

Substituting the expressions $\epsilon_{k_{j}+1}^{U}=-g_{j-1} \epsilon_{j-1}$ and $\epsilon_{k_{j}+1}^{V}=f_{j} \epsilon_{j-1}$ we get that

$$
\begin{aligned}
& \beta_{k_{j}+2}^{V}=-R_{j}\left(f_{j-1}-g_{j-1} \frac{f_{j}}{g_{j}}\right)=\frac{1}{g_{j}} R_{j}, \\
& \alpha_{k_{j}+2}^{V}=-N_{j}\left(f_{j-1}-g_{j-1} \frac{f_{j}}{g_{j}}\right)=\frac{1}{g_{j}} N_{j} .
\end{aligned}
$$

Thus if $h_{j+1}=1$ then $\epsilon_{k_{j}+2}^{V} \neq 0$, so Theorem 1 implies that

$$
\mu_{k_{j}+2}=\mu_{k_{j}+1}=l_{j}+1 \text { and } v_{k_{j}+2}=v_{k_{j}+1}+1=l_{j}+1 .
$$

This is the so-called generic case.

But if $h_{j+1} \geq 2$ then $\epsilon_{k_{j}+2}^{V}=0$ and the pair $N_{j}, R_{j}$ provides solution to the interpolation problem up to $2 l_{j}+h_{j+1}-1$. Theorem 4 implies that after that the controllability index $\mu$ remains constant and $v$ increases up to the point when they will be again equal. This happens at $k_{j+1}=2 l_{j+1}-1$. That is

$$
\mu_{k_{j+1}}=v_{k_{j+1}}=l_{j+1},
$$


concluding the proof of the theorem.

Funding Open Access funding provided by Eötvös Loránd University

Open Access This article is licensed under a Creative Commons Attribution 4.0 International License, which permits use, sharing, adaptation, distribution and reproduction in any medium or format, as long as you give appropriate credit to the original author(s) and the source, provide a link to the Creative Commons licence, and indicate if changes were made. The images or other third party material in this article are included in the article's Creative Commons licence, unless indicated otherwise in a credit line to the material. If material is not included in the article's Creative Commons licence and your intended use is not permitted by statutory regulation or exceeds the permitted use, you will need to obtain permission directly from the copyright holder. To view a copy of this licence, visit http://creativecommons.org/licenses/by/4.0/.

\section{Appendices}

\section{A Example: the Fibonacci sequence}

Let us consider the Fibonacci sequence of integers 1, 1, 2, 3, 5, 8, 13, 21, .. generated by the recursion $v_{j+1}=v_{j-1}+v_{j}$, with initial condition $v_{0}=1, v_{1}=1$. To make the computations more readable, we use the recursions (36) and (37) and we do not normalize.

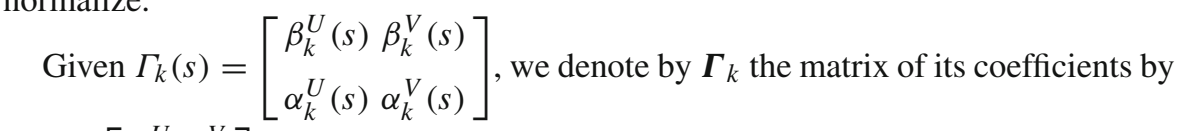
$\boldsymbol{\Gamma}_{k}:=\left[\begin{array}{cc}\boldsymbol{\beta}_{k}^{U} & \boldsymbol{\beta}_{k}^{V} \\ \boldsymbol{\alpha}_{k}^{U} & \boldsymbol{\alpha}_{k}^{V}\end{array}\right]$. So, the initial step is:

$$
\left[U_{0}^{1},-V_{0}^{0}\right] \boldsymbol{\Gamma}_{0}=\left[\begin{array}{ll}
1 & 0
\end{array}-1\right]\left[\begin{array}{ll}
0 & 1 \\
1 & 0 \\
\hline 0 & 1
\end{array}\right]=0, \quad \Gamma_{0}(s)=\left[\begin{array}{ll}
s & 1 \\
0 & 1
\end{array}\right]
$$

with the controllability indexes $\mu_{0}=1, v_{0}=0$. Adding one row of data $\left[u_{1}, u_{0},-v_{1}\right]$, we get

$$
\left[U_{1}^{1},-V_{1}^{0}\right] \boldsymbol{\Gamma}_{0}=\left[\begin{array}{ll|l}
1 & 0 & -1 \\
0 & 1 & -1
\end{array}\right]\left[\begin{array}{ll}
0 & 1 \\
1 & 0 \\
\hline 0 & 1
\end{array}\right]=\left[\begin{array}{cc}
0 & 0 \\
1 & -1
\end{array}\right]=\left[\begin{array}{cc}
0 & 0 \\
\epsilon_{1}^{U} & \epsilon_{1}^{V}
\end{array}\right]
$$

that is, $\epsilon_{1}^{V} \neq 0$ and $\mu_{0}>v_{0}$; we can thus increase the second controllability index $v_{0}$. Therefore, multiplying by $\left[\begin{array}{l}1 \\ 1\end{array}\right]$ we obtain an element in the kernel for the first column. We then add the column $-\mathcal{A}_{1} V_{1}=\left[\begin{array}{c}0 \\ -1\end{array}\right]$ to get $\left[U_{1}^{1},-V_{1}^{1}\right]$ and shift the second column of $\boldsymbol{\Gamma}_{0}$ to get $\boldsymbol{\Gamma}_{1}$ (the values of indexes are now $\mu_{1}=1, v_{1}=1$ ) and 
by construction

$$
\left[U_{1}^{1},-V_{1}^{1}\right] \boldsymbol{\Gamma}_{1}=\left[\begin{array}{cc|cc}
1 & 0 & -1 & 0 \\
0 & 1 & -1 & -1
\end{array}\right]\left[\begin{array}{ll}
1 & 0 \\
1 & 1 \\
\hline 1 & 0 \\
0 & 1
\end{array}\right]=0, \Gamma_{1}(s)=\left[\begin{array}{ccc}
s+1 & s \\
1 & s
\end{array}\right]
$$

The next row of data is $\left[u_{2}^{2},-v_{2}^{2}\right]=[0,0,-2,-1]$, and the errors are

$$
\left[\epsilon_{2}^{U}, \epsilon_{2}^{V}\right]=\left[u_{2}^{2},-v_{2}^{2}\right] \boldsymbol{\Gamma}_{1}=[-2,-1]
$$

Since $\epsilon_{2}^{U} \neq 0$, and $\mu_{1}=v_{1}=1$, we can thus set $\mu_{2}=\mu_{1}+1=2, \nu_{2}=v_{1}=1$, multiply $\boldsymbol{\Gamma}_{1}$ by $\left[\begin{array}{c}-1 \\ 2\end{array}\right]$ and obtain

$$
\left[U_{2}^{2},-V_{2}^{1}\right] \Gamma_{2}=\left[\begin{array}{lll|lc}
1 & 0 & 0 & -1 & 0 \\
0 & 1 & 0 & -1 & -1 \\
0 & 0 & 1 & -2 & -1
\end{array}\right]\left[\begin{array}{rr}
0 & -1 \\
1 & 1 \\
1 & 0 \\
\hline 0 & -1 \\
1 & 2
\end{array}\right]=0
$$

and

$$
\hat{\Gamma}_{2}(s)=\left[\begin{array}{cc}
s^{2}+s & s-1 \\
s & 2 s-1
\end{array}\right] .
$$

Now applying Theorem 1 we get that

$$
\Gamma_{2}(s)=\frac{1}{2}\left[\begin{array}{cc}
2 s^{2}+s+1 & s-1 \\
1 & 2 s-1
\end{array}\right] .
$$

A similar computation will yield $\boldsymbol{\Gamma}_{3}$ with errors $\left[\epsilon_{3}^{U}, \epsilon_{3}^{V}\right]=[-2,-1]$. Going up to $\boldsymbol{\Gamma}_{4}$, it is not hard to see that, for the data $\{1,1,2,3,5\}$, the new data row is $\left[u_{4}^{3},-v_{4}^{3}\right]=[0,0,0,-5,-3,-2]$ and the error is $[-1,-1]$. Thus, after some simple computations we obtain:

$$
\begin{gathered}
{\left[U_{4}^{3}, V_{4}^{2}\right] \boldsymbol{\Gamma}_{4}=\left[\begin{array}{llll|lcc}
1 & 0 & 0 & 0 & -1 & 0 & 0 \\
0 & 1 & 0 & 0 & -1 & -1 & 0 \\
0 & 0 & 1 & 0 & -2 & -1 & -1 \\
0 & 0 & 0 & 1 & -3 & -2 & -1 \\
0 & 0 & 0 & 0 & -5 & -3 & -2
\end{array}\right]\left[\begin{array}{rrr}
0 & -1 \\
2 & 0 \\
-1 & 0 \\
1 & 0 \\
\hline 0 & -1 \\
2 & 1 \\
-3 & 1
\end{array}\right]=0} \\
\hat{\Gamma}_{4}(s)=\left[\begin{array}{cccc}
s^{3}-s^{2}+2 s & -1 \\
-3 s^{2}+2 s & s^{2}+s-1
\end{array}\right]
\end{gathered}
$$


Applying again Theorem 1 we get that

$$
\Gamma_{4}(s)=\left[\begin{array}{cc}
s^{3}-s^{2}+2 s-3 & -1 \\
5 s-3 & s^{2}+s-1
\end{array}\right] \text {. }
$$

Considering the new interpolation value $v_{6}=8$ and thus adding the row $\left[u_{5}^{4},-v_{5}^{3}\right]=$ $[0,0,0,0,-8,-5,-3]$, we see that the errors are

$$
\left[\epsilon_{5}^{U}, \epsilon_{5}^{V}\right]=\left[u_{5}^{4},-v_{5}^{3}\right] \boldsymbol{\Gamma}_{4}=[-1,0] .
$$

Therefore, we cannot increase the index $\nu_{5}$. We thus have to increase $\mu_{5}$. While the Fibonacci sequence continues, this yields, for $k \geq 4, \Gamma_{k+1}(s)=\Gamma_{4}(s)\left[\begin{array}{cc}s^{k-3} & 0 \\ 0 & 1\end{array}\right]$. If for some $k_{0}$ the value $v_{k_{0}+1}$ is not a Fibonacci number (while all the previous are), then $\epsilon_{k_{0}+1}^{V} \neq 0$ and we can increase $v_{k_{0}}$ by multiplying the second column of $\Gamma_{k_{0}}(s)$ by $s$. A minimal interpolant will thus now have the degree of the first column of $\Gamma_{k_{0}+1}$, which is $k_{0}$.

\section{References}

1. Anderson BDO, Antoulas AC (1990) Rational interpolation and state-variable realizations. Linear Algebra Appl, vol 137/138. pp 479-509

2. Antoulas AC (1981) On canonical forms for linear constant systems. Int J Control 33:95-122

3. Antoulas AC, Ball JA, Kang J, Willems JC (1990) On the solution of the minimal rational interpolation problem. Linear Algebra Its Appl 137(138):511-573

4. Ball JA, Gohberg I, Rodman L (1988) Realization and interpolation of rational matrix functions. Oper Theory Adv Appl 33:1-72

5. Boros T, Sayed AH, Kailath T (1999) A recursive method for solving unconstrained tangential interpolation problems. IEEE-TAC 44(3):454-470

6. Dym H (1989) J-Contractive matrix functions, reproducing Kernel Hilbert spaces and interpolation, CBMS regional conference series in mathematics, vol 71. American Mathematical Society, Providence

7. Forney GD (1975) Minimal basis of rational vector spaces, with application to multivariable linear systems. SIAM J Control 13(3):493-520

8. Fuhrmann PA (2010) On tangential matrix interpolation. Linear Algebra Appl 433:2018-2059

9. Gombani A, Michaletzky G (2007) On interpolation and the Kimura-Georgiou parametrization. In: Chiuso A, Pinzoni S, Ferrante A (eds) Modeling, estimation and control, festschrift in honor of Giorgio Picci on the occasion of his sixty-fifth birthday, Springer lecture notes in control and information sciences, vol 364. Springer, Berlin, pp 171-182

10. Gragg WB, Lindquist A (1983) On the partial realisation problem. Linear Algebra Appl, vol 50. pp 277-319

11. Ho BL, Kalman R (1966) Effective construction of linear state-variable models from input/output functions. Regelungstechnik 14:545-548

12. Kailath T (1980) Linear systems. Prentice-Hall, Upper Saddle River

13. Kuijper M (1997) An algorithm for constructing a minimal partial realization in the multivariable case. Syst Control Lett 31:225-233

14. Lefteriu S, Antoulas A (2010) A new approach to modeling multiport systems from frequency-domain data. IEEE Trans Comput Aided Des Integr Circ Syst 29(1):14-27

15. Loewner K (1934) Über monotone Matrixfunctionen. Math Z 38:177-216

16. Mayo AJ, Antoulas AC (2007) a framework for the solution of the generalized realization problem. Linear Algebra Appl 425:634-662

17. Meijering E (2002) A chronology of interpolation. Proc IEEE 90:319-342 
18. Michaletzky G, Gombani A (2018) On multivariable proper rational interpolation using coprime factors. MCSS 30(2):1-37

19. Rissanen J (1971) Recursive identification of linear systems. SIAM J Control 9(3):420-430

20. Polderman JW, Willems JC (1998) Introduction to mathematical systems theory: a behavioral approach. Springer, New York

21. Wyman MF, Wyman BF (1985) An essay on continued fractions. Theory Comput Syst 18(1):295-328

Publisher's Note Springer Nature remains neutral with regard to jurisdictional claims in published maps and institutional affiliations. 